\title{
RESOLUTION OF SINGULARITIES AND GEOMETRIC PROOFS OF THE ŁOJASIEWICZ INEQUALITIES
}

\author{
PAUL M. N. FEEHAN
}

\begin{abstract}
The Łojasiewicz inequalities for real analytic functions on Euclidean space were first proved by Stanisław Łojasiewicz in [87, 88, 91] using methods of semianalytic and subanalytic sets, arguments later simplified by Bierstone and Milman [9]. Here, we first give an elementary geometric, coordinate-based proof of the Eojasiewicz inequalities in the special case where the function is $C^{1}$ with simple normal crossings. We then prove, partly following Bierstone and Milman [11, Section 2] and using resolution of singularities for (real or complex) analytic varieties, that the gradient inequality for an arbitrary analytic function follows from the special case where it has simple normal crossings. In addition, we prove the Łojasiewicz inequalities when a function is $C^{N}$ and generalized Morse-Bott of order $N \geq 3$; we gave an elementary proof of the Eojasiewicz inequalities when a function is $C^{2}$ and Morse-Bott on a Banach space in 36.
\end{abstract}

\section{INTRODUCTION}

Our goal is to provide geometric proofs of the Eojasiewicz inequalities (Theorem 1 and Corollaries 4 and 5) for functions with simple normal crossings and hence, via resolution of singularities, for arbitrary analytic functions on (real or complex) Euclidean space. In contrast, for a function that is (generalized) Morse-Bott (so its critical set is a submanifold), elementary methods suffice to prove the Łojasiewicz inequalities (Theorems 2.1 and 2.4).

The original proofs by Stanisław Łojasiewicz of his inequalities [89, 90, 91, 92, 94] relied on the theory of semianalytic sets and subanalytic sets originated by him and further developed by Gabrièlov [40, Hardt [54, 55] and Hironaka [63, 65, 62]. The proofs due to Łojasiewicz of his inequalities are well-known to be technically difficult. The most accessible modern approaches to the inequalities were provided by Bierstone and Milman. In [9], they significantly simplify the Łojasiewicz theory of semianalytic sets and subanalytic sets and prove his gradient inequality as a consequence of technical results in that theory. In [11, they develop an approach to resolution of singularities for algebraic and analytic varieties over a field of characteristic zero that relies on blowing up and greatly simplifies the original arguments due to Hironaka et al. [3, 4, 61, 64, They then deduce the Eojasiewicz gradient inequality as a consequence of resolution of singularities for analytic varieties and a direct verification when the critical and zero set of an analytic function is a simple normal crossing divisor.

Date: This version: January 6, 2020, incorporating final galley proof corrections. Geometry $\mathcal{E}$ Topology 23 (2019), no. 7, 3273-3313, https://doi.org/10.2140/gt.2019.23.3273 and https://arxiv.org/abs/1708.09775

2010 Mathematics Subject Classification. Primary 32B20, 32C05, 32C18, 32C25, 58E05; secondary 14E15, 32S45, 57R45, 58A07, 58A35.

Key words and phrases. Analytic varieties, Łojasiewicz inequalities, gradient flow, Morse-Bott functions, resolution of singularities, semianalytic sets and subanalytic sets.

The author was partially supported by National Science Foundation grant DMS-1510064 and the Simons Center for Geometry and Physics, Stony Brook, the Dublin Institute for Advanced Studies, the Institut des Hautes Études Scientifiques, Bures-sur-Yvette, and the Institute for Advanced Studies, Princeton. 
The Łojasiewicz gradient inequality was generalized by Leon Simon [112] to a certain class of real analytic functions on a Hölder space of $C^{2, \alpha}$ sections of a finite-rank vector bundle over a closed, finite-dimensional smooth manifold. Simon's proof relied on a splitting (or LyapunovSchmidt reduction) of the real analytic function into a finite-dimensional part, to which the original Łojasiewicz gradient inequality could be applied, and a benign infinite-dimensional part. The resulting Łojasiewicz-Simon gradient inequality and its many generalizations and variants have played a significant role in analyzing questions such as a) global existence, convergence, and analysis of singularities for solutions to nonlinear evolution equations that are realizable as gradient-like systems for an energy function, $b$ ) uniqueness of tangent cones, and $c$ ) gap theorems. See Feehan [34, Feehan and Maridakis [37, 38, and Huang 68, for references and a survey of Łojasiewicz-Simon gradient inequalities for analytic functions on Banach spaces and their many applications in applied mathematics, geometric analysis, and mathematical physics.

Our hope is that the more geometric and direct coordinate-based approaches provided in this article to proofs of the Łojasiewicz gradient inequality may yield greater insight that could be useful when endeavoring to prove gradient inequalities for functions on Banach spaces arising in geometric analysis without relying on Lyapunov-Schmidt reduction to the gradient inequality for functions on Euclidean space or attempting to extend methods specific to algebraic geometry. For example, the Łojasiewicz inequalities for the $F$ functional on the space of hypersurfaces in Euclidean space are proved directly by Colding and Minicozzi [25, 26, 27] and by the author for the Yang-Mills energy function near regular points in the moduli space of flat connections on a principal $G$-bundle over a closed, smooth Riemannian manifold [36]. Applications in geometric analysis typically concern functions on infinite-dimensional manifolds and, in that context, arguments specific to semianalytic sets or subanalytic subsets or real analytic subvarieties of Euclidean space do not necessarily have analogues in infinite-dimensional geometry. Like Bierstone and Milman in [11, Section 2], we ultimately apply resolution of singularities to obtain the Łojasiewicz gradient inequality for an arbitrary analytic function, but after directly proving the gradient inequality in simpler cases. When the function is $C^{N}$ and Morse-Bott of order $N \geq 2$, we obtain a Łojasiewicz exponent $\theta=1-1 / N$ (see Theorems 2.1 and 2.4) and when the function is $C^{1}$ with simple normal crossings, we obtain an explicit bound for the Łojasiewicz exponent which implies that $\theta \in[1 / 2,1)$ rather than $\theta \in(0,1)$ - together with a characterization of when $\theta$ has the optimal value $1 / 2$.

We showed in [36, Section 4] that one can use the Mean Value Theorem to prove the Eojasiewicz gradient inequality for a $C^{2}$ Morse-Bott function on a Banach space in a context of wide applicability [36, Theorem 3]. The facts that a Morse-Bott function has a critical set which is a smooth submanifold and a Hessian which is non-degenerate on the normal bundle ensure that the Mean Value Theorem easily yields the Łojasiewicz gradient inequality (with optimal Łojasiewicz exponent 1/2). In Section 3, we prove that the Eojasiewicz gradient inequality (Theorem 3) holds for a $C^{1}$ function that has simple normal crossings in the sense of Definition 1.1. We then appeal to resolution of singularities (Theorem 4.5) to show that the Eojasiewicz gradient inequality for an arbitrary analytic function, Theorem 1, is a straightforward consequence of Theorem 3 . This incremental approach makes it clear that the essential difficulty is due neither to the high dimension of the ambient Euclidean space nor the critical set, but instead due (as should be expected) to possibly complicated singularities in the critical set.

Simplifications of Łojasiewicz's proofs [91] of his inequalities have also been given by Kurdyka and Parusiński [79, where they use the fact that a subanalytic set in Euclidean space admits a strict Thom stratification. Łojasiewicz and Zurro [95] further simplified the arguments of Kurdyka and Parusiński to prove the Łojasiewicz inequalities, again using properties of subanalytic sets. 
The problem of estimating Łojasiewicz exponents or determining their properties, often for restricted classes of functions (for example, polynomials, certain analytic functions, functions with isolated critical points, and so on), has been pursued by many researchers, including Abderrahmane [1, Bivià-Ausina [13, Bivià-Ausina and Encinas [14, 15, 16], Bivià-Ausina and Fukui [17, Brzostowski [20], Brzostowski, Krasiński, and Oleksik [21], Búi and Pham [22], D'Acunto and Kurdyka [30], Fukui [39, Gabrièlov [41] Gwoździewicz [47], Haraux [48, Theorem 3.1], Haraux and Pham [52, 53], Ji, Kollár, and Shiffman [69] Kollár [72], Krasiński, Oleksik, and Płoski [75], Kuo [77, Lichtin [85], Lenarcik [82, 83], Lion [86], Oka [98], Oleksik [99], Pham [101, 102], Płoski [103, Risler and Trotman [104, Rodak and Spodzieja [105], 117], Tan, Yau, and Zuo [118, and Teissier [119. Recently, simpler coordinate-based proofs of more limited versions of resolution of singularities for zero sets of real analytic functions, with applications to analysis, have been given by Collins, Greenleaf, and Pramanik [28] and Greenblatt [44. In particular, Greenblatt [44, p. 1959] applies his version of resolution of singularities to prove the Eojasiewicz inequality (1.2) for a pair of real analytic functions where the zero set of one is contained in the zero set of the other. Bivià-Ausina and Encinas [14] use a resolution of singularities algorithm to estimate Łojasiewicz exponents.

Łojasiewicz [87, 88] applied his distance inequality (Corollary 44) to prove the Division Conjecture of Schwartz [108, p. 181], [109, p. 116]. In [89], he used his gradient inequality (Theorem 11) to give a positive answer [89, Theorem 5] to a question of Whitney: If $\mathscr{E}$ is a real analytic function on an open set $U \subset \mathbb{R}^{d}$, then $\mathscr{E}^{-1}(0)$ is a deformation retract of its neighborhood. This deformation retract is obtained using the negative gradient flow defined by $\mathscr{E}$. He also applies his inequalities to show that every (locally closed) semianalytic set in Euclidean space admits a Whitney stratification 1 [91, Proposition 3, p. 97 (71)]. The Eojasiewicz gradient inequality (Theorem 11) was used by Kurdyka, Mostowski, and Parusiński [78] to prove the Gradient Conjecture of Thom.

Atiyah [5] and Bernstein and Gelfand [8] appear to be the first authors to have noticed that resolution of singularities could be used to simplify proofs of Łojasiewicz's results, a fact that we discovered only when correcting galley proofs for this article. In [5], Atiyah employed resolution of singularities to give a simple proof of the Division Conjecture, using methods similar to those in our proof of Theorem 1. Atiyah notes [5, p. 145] that Bernstein and Gelfand independently proved the Division Conjecture using related ideas in [8]. The only article that is firmly in the literature on Łojasiewicz inequalities that cites Atiyah is due to Bivià-Ausina and Fukui [17].

1.1. Main results. We let $\mathbb{K}=\mathbb{R}$ or $\mathbb{C}$ and state the main results to be proved in this article, categorized according to whether or not their proofs appeal to resolution of singularities.

\subsubsection{Gradient inequality using resolution of singularities. We begin with the fundamental}

Theorem 1 (Łojasiewicz gradient inequality for an analytic function). (See Eojasiewicz 91, Proposition 1, p. 92 (67)].) Let $d \geq 1$ be an integer, $U \subset \mathbb{K}^{d}$ be an open subset, and $\mathscr{E}: U \rightarrow \mathbb{K}$ be an analytic function. If $x_{\infty} \in U$ is a point such that $\mathscr{E}^{\prime}\left(x_{\infty}\right)=0$, then there are constants $C_{0} \in(0, \infty)$, and $\sigma_{0} \in(0,1]$, and $\theta \in[1 / 2,1)$ such that the differential map, $\mathscr{E}^{\prime}: U \rightarrow \mathbb{K}^{d *}$, obeys

$$
\left\|\mathscr{E}^{\prime}(x)\right\|_{\mathbb{K}^{d *}} \geq C_{0}\left|\mathscr{E}(x)-\mathscr{E}\left(x_{\infty}\right)\right|^{\theta}, \quad \text { for all } x \in B_{\sigma_{0}}\left(x_{\infty}\right),
$$

where $\mathbb{K}^{d *}=\left(\mathbb{K}^{d}\right)^{*}$, the dual space of $\mathbb{K}^{d}$ and $B_{\sigma_{0}}\left(x_{\infty}\right):=\left\{x \in \mathbb{K}^{d}:\left\|x-x_{\infty}\right\|_{\mathbb{K}^{d}}<\sigma_{0}\right\} \subset U$.

\footnotetext{
${ }^{1}$ The first page number refers to the version of Łojasiewicz's original manuscript mimeographed by IHES while the page number in parentheses refers to the cited LaTeX version of his manuscript prepared by M. Coste and available on the Internet.
} 
By definition, the Lojasiewicz exponent $\theta$ of a $C^{1}$ function $\mathscr{E}$ at a point $x_{\infty}$ in its domain is the smallest $\theta \geq 0$ such that the inequality (1.1) holds for some positive constant $C_{0}$ and all $x$ in an open neighborhood of $x_{\infty}$.

Theorem 11was stated by Łojasiewicz in [89, Theorem 4] and proved by him as [91, Proposition 1, p. 92]; see also Łojasiewicz [94, p. 1592]. Bierstone and Milman provided simplified proofs as [9, Proposition 6.8] and [11, Theorem 2.7]. Their strategy in [9] is to first prove a Łojasiewicz inequality [9, Theorem 6.4] of the form

$$
|g(x)| \geq C|f(x)|^{\lambda}, \quad \text { for all } x \in B_{\sigma},
$$

where $f$ and $g$ are subanalytic functions on an open neighborhood $U \subset \mathbb{R}^{d}$ of the origin such that $g^{-1}(0) \subset f^{-1}(0)$ and $B_{\sigma} \subset U$ and $\lambda \in(0, \infty)$. They then deduce a Łojasiewicz gradient inequality [9, Theorem 6.8] for a real analytic function $f$ with $f^{\prime}(0)=0$,

$$
\left\|f^{\prime}(x)\right\|_{\mathbb{R}^{d *}} \geq C|f(x)|^{\nu}, \quad \text { for all } x \in B_{\sigma},
$$

with $\nu \in(0,1)$ by choosing $g=\left\|f^{\prime}\right\|_{\mathbb{R}^{d *}}$. In [11, Theorem 2.5], the authors establish (1.2) for a pair of (real or complex) analytic functions by using resolution of singularities to reduce to the case that the ideal in the ring of analytic functions, $\mathscr{O}_{X}$, generated by $f g$ has simple normal crossings. In [11, Theorem 2.7], they then obtain (1.3) for an analytic function $f$ with $f(0)=0$ and $f^{\prime}(0)=0$ by choosing $g=\left\|f^{\prime}\right\|_{\mathbb{K} d *}^{2}$ and applying (1.2) to the pair of functions $f^{2}$ (replacing $g$ ) and $f^{2} / g$ (replacing $f$ ) and proving that $f^{-1}(0) \subset\left(f^{2} / g\right)^{-1}(0)$ and $\nu=1 / \lambda \in(0,1)$, after employing resolution of singularities to the ideal $f g \mathscr{O}_{X}$.

Our more direct proof of Theorem 1 makes it clear that one always has $\theta \geq 1 / 2$, whereas previous proofs only gave $\theta \in(0,1)$. For applications to geometric analysis and topology, it is essential to have $\theta<1$, with $\theta=1 / 2$ being the optimal exponent, corresponding to exponential convergence for the negative gradient flow defined by $\mathscr{E}$. In particular, we have:

Corollary 2 (Characterization of the optimal exponent and Morse-Bott condition). Assume the hypotheses of Theorem 1 and that $x_{\infty}$ is the origin. If $\theta=1 / 2$ then, after possibly shrinking $U$, there are an open neighborhood of the origin, $\widetilde{U} \subset \mathbb{K}^{d}$, and an analytic map, $\pi: \widetilde{U} \rightarrow U$, such that $\pi$ is an analytic diffeomorphism on the complement of a coordinate hyperplane or the union of two coordinate hyperplanes and $\pi^{*} \mathscr{E}$ is Morse-Bott at the origin in the sense of Definition 1.2.

See the author's [34, Theorem 3] for the statement and proof of a very general convergence-rate result for an abstract gradient flow on a Banach space defined by an analytic function obeying a Łojasiewicz-Simon gradient inequality with exponent $\theta \in[1 / 2,1)$ and for previous versions of related convergence-rate results, see Chill, Haraux, and Jendoubi [24, Theorem 2], Haraux, Jendoubi, and Kavian [51, Propositions 3.1 and 3.4], Huang [68, Theorem 3.4.8], and Råde [107, Proposition 7.4]. Convergence-rate results related to [34, Theorem 3] are implicit in Adams and Simon [2] and Simon [112, 113, 114, although we cannot find an explicit statement like this in those references.

1.1.2. Gradient inequality without using resolution of singularities. The proof of Theorem 1 in full generality provided in this article employs embedded resolution of singularities (partly following Bierstone and Milman [11, Section 2]), but there are several weaker gradient inequalities that can be proved by far more elementary methods and those provide insight to applications in geometric analysis. We now describe several results of this kind. For example, when the function $\mathscr{E}$ in Theorem 1 is $C^{2}$ (respectively, $C^{N}$ with $N \geq 2$ ) and Morse-Bott (respectively, Morse-Bott of order $N$ ), rather than an arbitrary analytic function, one obtains the Eojasiewicz gradient inequality with exponent $\theta=1 / 2$ (respectively, $\theta=1-1 / N$ ) as a consequence of the Mean 
Value Theorem (respectively, Taylor Theorem): see Theorems 2.1 and 2.4. We refer the reader to Section 2 for a discussion of the Morse-Bott condition and some its generalizations, together with the statements and proofs of Theorems 2.1 and 2.4 .

A first reading of the proof of Theorem 2.4, which is based on a direct application of the Taylor Theorem, might suggest that it would extend to the case where $\mathscr{E}$ is an analytic function and $U \cap$ Crit $\mathscr{E}$ is an arbitrary analytic subvariety. However, one finds that this is a more difficult strategy to develop than one might naively expect. Instead, as a stepping stone towards Theorem 1. we shall first establish a special case that holds for a class of $C^{1}$ functions. By analogy with Collins, Greenleaf, and Pramanik [28, Definition 2.5], we make the

Definition 1.1 (Function with simple normal crossings). A $C^{1}$ function $f: U \rightarrow \mathbb{K}$ on an open neighborhood of the origin in $\mathbb{K}^{d}$ has simple normal crossings if

$$
f(x)=x_{1}^{n_{1}} \cdots x_{d}^{n_{d}} f_{0}(x), \quad \text { for all } x=\left(x_{1}, \ldots, x_{d}\right) \in U,
$$

where $n_{i} \in \mathbb{Z} \cap[0, \infty)$ and $f_{0}$ is a $C^{1}$ function such that $f_{0}(0) \neq 0$ and $N=\sum_{i=1}^{d} n_{i}$ is the total degree of the monomial $x_{1}^{n_{1}} \cdots x_{d}^{n_{d}}$.

See Sections 4.1 and 4.2 for a review of normal crossings and simple normal crossings divisors in (real or complex) analytic geometry.

Definition 1.2 (Morse-Bott function). Let $d \geq 1$ be an integer, $U \subset \mathbb{K}^{d}$ be an open subset, $\mathscr{E}: U \rightarrow \mathbb{K}$ be a $C^{2}$ function, and Crit $\mathscr{E}:=\left\{x \in U: \mathscr{E}^{\prime}(x)=0\right\}$. We say that $\mathscr{E}$ is Morse-Bott at a point $x_{\infty} \in$ Crit $\mathscr{E}$ if $a$ ) Crit $\mathscr{E}$ is a $C^{2}$ submanifold of $U$, and $\left.b\right) T_{x_{\infty}} \operatorname{Crit} \mathscr{E}=\operatorname{Ker} \mathscr{E}^{\prime \prime}\left(x_{\infty}\right)$ when $\mathscr{E}^{\prime \prime}\left(x_{\infty}\right)$ is considered as an operator in $\operatorname{Hom}_{\mathbb{K}}\left(\mathbb{K}^{d}, \mathbb{K}^{d *}\right)$, where $T_{x}$ Crit $\mathscr{E}$ is the tangent space to Crit $\mathscr{E}$ at a point $x \in$ Crit $\mathscr{E}$.

In applications to topology (see, for example, Austin and Braam [6, Section 3.1] for equivariant Floer cohomology and Bott [19] for the Periodicity Theorem), our local Definition [1.2 is often augmented by conditions that Crit $\mathscr{E}$ be compact, as in Bott [18, Definition, p. 248], or compact and connected as in Nicolaescu [97, Definition 2.41], and that $T_{x} \operatorname{Crit} \mathscr{E}=\operatorname{Ker} \mathscr{E}^{\prime \prime}(x)$ for all $x \in$ Crit $\mathscr{E}$.

Theorem 3 (Eojasiewicz gradient inequality for a $C^{1}$ function with simple normal crossings and characterization of the optimal exponent and Morse-Bott condition). Let $d \geq 2$ be an integer, $U \subset \mathbb{K}^{d}$ be an open neighborhood of the origin, and $\mathscr{E}: U \rightarrow \mathbb{K}$ be a $C^{1}$ function with simple normal crossings. If $\mathscr{E}^{\prime}(0)=0$, then the followibng hold.

(1) There are constants $C_{0} \in(0, \infty)$ and $\sigma \in(0,1]$ such that

$$
\left\|\mathscr{E}^{\prime}(x)\right\|_{\mathbb{K}^{d *}} \geq C_{0}|\mathscr{E}(x)|^{\theta}, \quad \text { for all } x \in B_{\sigma},
$$

where $\theta=1-1 / N \in[1 / 2,1)$ and $N=\sum_{i=1}^{d} n_{i}$ is the total degree of the monomial in the expression (1.4) for $\mathscr{E}$.

(2) If $c$ is the number of exponents $n_{i} \geq 1$ for $i=1, \ldots, d$, then $c \geq 2$ or $c=1$ and (after relabeling coordinates) $n_{1} \geq 2$.

(3) One has $\theta=1 / 2$ if and only if $c=2$ and (after relabeling coordinates) $n_{1}=n_{2}=1$ or $c=1$ and $n_{1}=2$.

(4) If $\theta=1 / 2$ and $\mathscr{E}$ is $C^{2}$, then $\mathscr{E}$ is Morse-Bott on $B_{\sigma}$.

Remark 1.3 (Geometry of the critical set). Theorem 2.1 shows that, when $\mathscr{E}$ is Morse-Bott and so its critical set is a smooth submanifold, then its Łojasiewicz exponent $\theta$ is equal to $1 / 2$. Conversely, when $\theta=1 / 2$, Theorem 3 implies that $B_{\sigma} \cap$ Crit $\mathscr{E}=\left\{x_{1}=0\right\} \cap B_{\sigma}$ or $\left\{x_{1}=x_{2}=0\right\} \cap B_{\sigma}$, a 
codimension-one or codimension-two smooth submanifold of $B_{\sigma}$. Theorem 1 is proved by applying resolution of singularities to an ideal defined by an arbitrary analytic function $\mathscr{E}$ and applying Theorem 3 to the resulting monomial (the product of $x_{1}^{n_{1}} \cdots x_{d}^{n_{c}}$ and a non-vanishing analytic function). Consequently, if $\theta=1 / 2$ then there is a constraint on the nature of the singularities in the critical set of $\mathscr{E}$. Our proof of Theorem 1 shows that application of resolution of singularities does not change the Łojasiewicz exponent and so it is of interest to try to characterize the class of analytic functions with $\theta=1 / 2$, a topic that we explore in Feehan [35]. As noted in our Introduction, the problem of computing or estimating Łojasiewicz exponents remains a topic of active research.

Our proof of Theorem 3 is a direct coordinate-based alternative to an argument due to Bierstone and Milman [11, Section 2] and relies only on the Generalized Young Inequality (3.7) (see Remark (3.1)). We are grateful to Alain Haraux for pointing out that the value for $\theta$ in previous versions of this article could be improved to the value now stated in Theorem 3 and for alerting us to his [48, Theorem 3.1]. His result is more closely related to Theorem 3 than we had realized (it assumes $f_{0}=1$ in the expression (1.4)) and we were unaware that his proof also used the Generalized Young Inequality.

1.1.3. Consequences of the gradient inequality. Regardless of how proved, the gradient inequality (1.1) easily yields two useful corollaries. Note that if $\mathscr{E}(x)$ is differentiable at $x=x_{0}$ and $\mathscr{E}\left(x_{0}\right)=0$, then $\mathscr{E}(x)^{2}$ has a critical point at $x=x_{0}$. We say that a subset $A \subset \mathbb{K}^{d}$ is $C^{k}$ arc connected if any two points in $A$ can be joined by a $C^{k}$ curve, where $k \in \mathbb{Z} \cap[0, \infty)$ or $k=\omega$ (analytic), and locally $C^{k}$-arc connected if for every point $x \in A$ has an open neighborhood $U \subset \mathbb{K}^{d}$ such that $U \cap A$ is $C^{k}$-arc connected.

Corollary 4 (Łojasiewicz distance inequalities). (Compare Eojasiewicz [91, Theorem 2, p. 85 (62)].) Let $d \geq 1$ be an integer, $U \subset \mathbb{R}^{d}$ be an open neighborhood of the origin and $\mathscr{E}: U \rightarrow \mathbb{R}$ be a $C^{1}$ function.

(1) (Distance to the critical and zero sets.) If $\mathscr{E}(0)=0$ and $\mathscr{E}^{\prime}(0)=0$ and $\mathscr{E} \geq 0$ on $U$ and $\mathscr{E}$ obeys the Eojasiewicz gradient inequality (1.1) near the origin, then there are constants $C_{1} \in(0, \infty)$, and $\delta \in(0, \sigma / 4]$, and $\alpha=1 /(1-\theta) \in[2, \infty)$ such that

$$
\mathscr{E}(x) \geq C_{1} \operatorname{dist}_{\mathbb{R}^{d}}\left(x, B_{\sigma} \cap \text { Crit } \mathscr{E}\right)^{\alpha}, \quad \text { for all } x \in B_{\delta},
$$

where $\operatorname{dist}_{\mathbb{R}^{d}}(x, A):=\inf \left\{\|x-a\|_{\mathbb{R}^{d}}: a \in A\right\}$, for any point $x \in \mathbb{R}^{d}$ and subset $A \subset \mathbb{R}^{d}$. If in addition $B_{\sigma} \cap$ Crit $\mathscr{E} \subset B_{\sigma} \cap$ Zero $\mathscr{E}$, then

$$
\mathscr{E}(x) \geq C_{1} \operatorname{dist}_{\mathbb{R}^{d}}\left(x, B_{\sigma} \cap \text { Zero } \mathscr{E}\right)^{\alpha}, \quad \text { for all } x \in B_{\delta},
$$

where Zero $\mathscr{E}:=\{x \in U: \mathscr{E}(x)=0\}$.

(2) (Distance to the zero set.) If $\mathscr{E}(0)=0$ and $\mathscr{E}^{2}$ (in place of $\mathscr{E}$ ) obeys the Lojasiewicz gradient inequality (1.1) near the origin and $B_{\sigma} \cap$ Crit $\mathscr{E}^{2} \subset B_{\sigma} \cap$ Zero $\mathscr{E}$, then there are constants $C_{2} \in(0, \infty)$, and $\delta \in(0, \sigma / 4]$, and $\beta=1 /(2(1-\theta)) \in[1, \infty)$ such that

$$
|\mathscr{E}(x)| \geq C_{2} \operatorname{dist}_{\mathbb{R}^{d}}\left(x, B_{\sigma} \cap \text { Zero } \mathscr{E}\right)^{\beta}, \quad \text { for all } x \in B_{\delta} .
$$

Remark 1.4 (Analytic functions obey the hypotheses of Corollary 4 ). If $\mathscr{E}$ is analytic, then the hypotheses in Corollary 4 that $\mathscr{E}$ or $\mathscr{E}^{2}$ obey (1.1) are implied by Theorem 1 .

Moreover, if $\mathscr{E}$ is analytic, then Crit $\mathscr{E}$ and Crit $\mathscr{E}^{2}$ are analytic subvarieties of $U$ and thus locally connected by [9, Corollary $2.7(3)]$ and hence locally $C^{0}$-arc connected by [74, Exercise 29F]. By Gabriélov [40, p. 283], analytic subvarieties of $U$ are locally analytic-arc connected and so Crit $\mathscr{E}$ and Crit $\mathscr{E}^{2}$ are locally $C^{1}$-arc connected by [40, p. 283] when $\mathscr{E}$ is analytic. In 
particular, if Crit $\mathscr{E}$ is locally $C^{1}$-arc connected, then $B_{\sigma} \cap$ Crit $\mathscr{E} \subset B_{\sigma} \cap$ Zero $\mathscr{E}$, as assumed in the second half of Item (1); if Crit $\mathscr{E}^{2}$ is locally $C^{1}$-arc connected, then $B_{\sigma} \cap$ Crit $\mathscr{E}^{2} \subset B_{\sigma} \cap$ Zero $\mathscr{E}$, as assumed in Item (2).

When $\mathscr{E}$ is analytic, Item (2) in Corollary 4 was stated by Łojasiewicz in [89, Corollary, p. 88] and proved by him in [87, Theorem 17, p. 124], [88, Theorem 17, p. 40]; it was restated and proved by him as [91, Theorem 2, p. 85 (62)]. Simplified proofs of Item (2) in Corollary 4 were provided by Bierstone and Milman as [9, Theorem 6.4 and Remark 6.5] and [11, Theorem 2.8]. When $\mathscr{E}$ is a polynomial on $\mathbb{R}^{d}$, Corollary 4 is due to Hörmander [67, Lemma 1]. The next result is obtained by combining Theorem 1 and Item (10) in Corollary 4 .

Corollary 5 (Łojasiewicz gradient-distance inequality for a non-negative function). Let $d \geq 1$ be an integer, $U \subset \mathbb{R}^{d}$ be an open neighborhood of a point $x_{\infty}$, and $\mathscr{E}: U \rightarrow \mathbb{R}$ be a $C^{1}$ function. If $\mathscr{E}^{\prime}\left(x_{\infty}\right)=0$ and $\mathscr{E} \geq 0$ on $U$ and $\mathscr{E}$ obeys the Eojasiewicz gradient inequality (1.1) near $x_{\infty}$, then there are constants $C_{2} \in(0, \infty)$, and $\delta \in(0, \sigma / 4]$, and $\mu=\theta /(1-\theta) \in[1, \infty)$ such that

$$
\left\|\mathscr{E}^{\prime}(x)\right\|_{\mathbb{R}^{d *}} \geq C_{2} \operatorname{dist}\left(x, B_{\sigma} \cap \operatorname{Crit} \mathscr{E}\right)^{\mu}, \quad \text { for all } x \in B_{\delta}\left(x_{\infty}\right) .
$$

When $\mathscr{E}$ is analytic, the hypothesis in Corollary $[5$ that $\mathscr{E} \geq 0$ on $U$ can be relaxed.

Corollary 6 (Łojasiewicz gradient-distance inequality for an analytic function). Let $d \geq 1$ be an integer, $U \subset \mathbb{R}^{d}$ be an open neighborhood of a point $x_{\infty}$, and $\mathscr{E}: U \rightarrow \mathbb{R}$ be an analytic function. If $\mathscr{E}^{\prime}\left(x_{\infty}\right)=0$, then there are constants $C_{3} \in(0, \infty)$, and $\sigma_{1} \in(0,1]$, and $\delta_{1} \in\left(0, \sigma_{1} / 4\right]$, and $\gamma \in[1 / 2, \infty)$ such that

$$
\left\|\mathscr{E}^{\prime}(x)\right\|_{\mathbb{R}^{d *}} \geq C_{3} \operatorname{dist}_{\mathbb{R}^{d}}\left(x, B_{\sigma_{1}} \cap \operatorname{Crit} \mathscr{E}\right)^{\gamma}, \quad \text { for all } x \in B_{\delta_{1}}\left(x_{\infty}\right) .
$$

The inequality (1.10) is stated by Simon in [112, Equation (2.3)] and attributed by him to Łojasiewicz [91].

1.1.4. Counterexamples. It is known but worth remembering that the Eojasiewicz gradient inequality fails in general for functions that are smooth but not analytic. For example, De Lellis [31] notes that when $d=1$, then the function

$$
\mathscr{E}(x)= \begin{cases}e^{1 /|x|}, & x \neq 0, \\ 0, & x=0,\end{cases}
$$

is $C^{\infty}$ on $\mathbb{R}$ with Crit $\mathscr{E}=\{0\}$ but that the inequality (1.1) fails on any open neighborhood of the origin. When $d=2$ and $\mathbb{K}=\mathbb{R}$, Haraux shows in [48, Proposition 5.2] that for the $C^{1}$ function,

$$
\mathscr{E}(x, y)= \begin{cases}\left(x^{2}+y^{2}\right) e^{-\left(x^{2}+y^{2}\right) / x^{2}}, & x \neq 0, \\ 0, & x=0,\end{cases}
$$

the inequality (1.1) fails on any neighborhood of the origin. Moreover, failure of a smooth function to satisfy the Łojasiewicz gradient inequality may result in non-convergence of its negative gradient flow: see Haraux [48, Remark 5.5] (citing Palis and de Melo [100]), Haraux and Jendoubi [50, Section 12.8], and Lerman [84] (citing [100, p. 14]).

1.2. Outline. We begin in Section[2 with elementary proofs of the Eojasiewicz gradient inequality for functions that are Morse-Bott (Theorem 2.11) or generalized Morse-Bott (Theorem 2.4). In Section 3, we establish the Łojasiewicz gradient inequality (Theorem 3) for $C^{1}$ functions with simple normal crossings. In Section 4 , we review the resolution of singularities for analytic varieties (Theorem 4.5) and apply that and Theorem 3 to prove the Eojasiewicz gradient inequality for an arbitrary analytic function (Theorem 1). Finally, in Section 5 we deduce Corollaries 4 . 
5. and 6 from the gradient inequality (1.1). Appendix $\mathrm{A}$ illustrates the application of resolution of singularities (Theorem 4.5) to achieve the required monomialization in the case of a simple example, namely the cusp curve.

1.3. Acknowledgments. I thank Kenji Fukaya for his description of an example that alerted me to an error in an entirely different approach described in an early draft of this article. I am grateful to Lev Borisov, Tristan Collins, Antonella Grassi, Michael Greenblatt, Johan de Jong, Peter Kronheimer, Claude LeBrun, Tom Mrowka, Graeme Wilkin, and Jarek Włodarczyk for helpful communications or discussions, to Carles Bivià-Ausina, Santiago Encinas, Alain Haraux and Dennis Sullivan for many helpful comments and suggestions, to Manousos Maridakis for engaging conversations regarding Łojasiewicz inequalities, to Toby Colding and Mark Goresky for their interest in this work, and to our librarian at Rutgers, Mei-Ling Lo, for locating difficultto-find articles on my behalf. I am indebted to Tomáš Bárta for helpful discussions and especially for pointing out an example (see Remark 2.3) showing that a previous version of my Definition 2.3 in an older version of this article was incomplete. I am grateful to the National Science Foundation (grant DMS-1510064) for their support and to the Simons Center for Geometry and Physics, Stony Brook, the Dublin Institute for Advanced Studies, the Institut des Hautes Études Scientifiques, Bures-sur-Yvette, and the Institute for Advanced Studies, Princeton, for their hospitality and support during the preparation of this article. Lastly, I thank the anonymous referees for their careful reading of our manuscript and for their suggestions.

\section{2. Łojasiewicz Gradient inequalities for Generalized Morse-Bott Functions}

In this section, we adapt our previous proof in [36] of the Eojasiewicz inequalities for MorseBott functions on Banach spaces [36, Theorem 3] (restated here as Theorem 2.1 for the case of Euclidean spaces) to prove the Eojasiewicz gradient inequality for generalized Morse-Bott functions, namely Theorem 2.4, our [36. Theorem 3] improves upon [37, Theorems 3 and 4] and has a simpler proof. Theorem 2.1 was proved by Simon [114, Lemma 3.13.1] (for a harmonic map energy function on a Banach space of $C^{2, \alpha}$ sections of a Riemannian vector bundle), Haraux and Jendoubi [49, Theorem 2.1] (for functions on abstract Hilbert spaces), and in greater generality by Chill in [23. Corollary 3.12] (for functions on abstract Banach spaces); a more elementary version was proved by Huang as [68, Proposition 2.7.1] (for functions on abstract Banach spaces). These authors do not use Morse-Bott terminology but their hypotheses imply this condition directly in the case of Haraux and Jendoubi and Chill and by a remark due to Simon in [114, p. 80] that his integrability condition [114, Equation (iii), p. 79] is equivalent to a restatement of the Morse-Bott condition. See Feehan [35] for further discussion of the relationship between definitions of integrability, such as those described by Adams and Simon [2], and the Morse-Bott condition.

2.1. Morse-Bott and generalized Morse-Bott functions. We begin with a well-known result.

Theorem 2.1 (Łojasiewicz gradient inequality for a Morse-Bott function on Euclidean space). Let $d \geq 1$ be an integer and $U \subset \mathbb{K}^{d}$ an open subset. If $\mathscr{E}: U \rightarrow \mathbb{K}$ is a Morse-Bott function, then there are constants $C_{0} \in(0, \infty)$ and $\sigma_{0} \in(0,1]$ such that

$$
\left\|\mathscr{E}^{\prime}(x)\right\|_{\mathbb{K}^{d *}} \geq C_{0}\left|\mathscr{E}(x)-\mathscr{E}\left(x_{\infty}\right)\right|^{1 / 2}, \quad \text { for all } x \in B_{\sigma_{0}}\left(x_{\infty}\right) .
$$

Theorem 2.1 is a special case of Feehan [36, Theorem 3] and Feehan and Maridakis [37, Theorems 3 and 4], where the case of a Morse-Bott function on a Banach space is considered. 
Even when $\mathscr{E}$ is a Morse-Bott function on a Banach space, the proof of the corresponding Łojasiewicz gradient inequality [36, Theorem 3] still readily follows from the Mean Value Theorem (see [36, Section 4]) in the presence of a few additional technical hypotheses specific to the infinite-dimensional setting.

Remark 2.2 (On the proof of Theorem 2.1). The conclusion of Theorem 2.1) is a simple consequence of the Morse-Bott Lemma (see Banyaga and Hurtubise [7, Theorem 2], Nicolaescu [97, Proposition 2.42], or Feehan [35]). However, the proof of the Morse-Bott Lemma itself (especially for MorseBott functions that are at most $C^{2}$ ) requires care. In contrast, our proof of Theorem 2.1 - given as the proof of [36. Theorem 3] in the infinite-dimensional case - is direct and elementary and avoids appealing to the Morse-Bott Lemma.

Definition 2.3 (Generalized Morse-Bott function). Let $d \geq 1$ and $N \geq 2$ be integers, $U \subset \mathbb{K}^{d}$ be an open subset, and $\mathscr{E}: U \rightarrow \mathbb{K}$ be a $C^{N}$ function. We call $\mathscr{E}$ a generalized Morse-Bott function of order $N$ at a point $x_{\infty} \in$ Crit $\mathscr{E}$ if $(a)$ Crit $\mathscr{E}$ is a $C^{N}$ submanifold of $U,(b) \mathscr{E}^{(n)}(x)=0$ for all $x \in \operatorname{Crit} \mathscr{E}$ and $1 \leq n \leq N-1$, and $(c) \mathscr{E}^{(N)}\left(x_{\infty}\right) \xi^{N} \neq 0$ for all nonzero $\xi \in T_{x_{\infty}}^{\perp}$ Crit $\mathscr{E}$, where $T_{x_{\infty}}^{\perp}$ Crit $\mathscr{E}$ is the orthogonal complement of $T_{x_{\infty}}$ Crit $\mathscr{E}$ in $\mathbb{K}^{d}$.

For example, if $N \geq 2$ and $f(x, y)=x^{N}$ then $f: \mathbb{K}^{2} \rightarrow \mathbb{K}$ is a generalized Morse-Bott function of order $N$. The analogous definition of a generalized Morse function is stated, for example, by Rothe [106, Definition 2.6] and Kuiper [76, p. 202, Corollary]. While Definition 2.3 is valid when $N=2$, the conditions are then more restrictive than those of Definition 1.2,

Theorem 2.4 (Eojasiewicz gradient inequality for a generalized Morse-Bott function on Euclidean space). Let $d \geq 1$ and $N \geq 2$ be integers and $U \subset \mathbb{K}^{d}$ be an open neighborhood. If $\mathscr{E}: U \rightarrow \mathbb{K}$ is a generalized Morse-Bott function of order $N$ at a point $x_{\infty} \in$ Crit $\mathscr{E}$, then there are constants $C_{0} \in(0, \infty)$ and $\sigma_{0} \in(0,1]$ such that

$$
\left\|\mathscr{E}^{\prime}(x)\right\|_{\mathbb{K}^{d *}} \geq C_{0}\left|\mathscr{E}(x)-\mathscr{E}\left(x_{\infty}\right)\right|^{1-1 / N}, \quad \text { for all } x \in B_{\sigma_{0}}\left(x_{\infty}\right) .
$$

As Definition 2.3 suggests, the proof of Theorem 2.4 should generalize to the setting of functions on Banach spaces, as in [36, Theorem 3] for the case of Morse-Bott functions.

Remark 2.5 (Comparison of Theorem 2.1 and Theorem 2.4 when $N=2$ ). While Theorem 2.4 holds when $N=2$, Theorem 2.1 is a stronger result since condition (b) in Definition 1.2, which is equivalent to the condition that $\mathscr{E}^{\prime \prime}\left(x_{\infty}\right) \in \operatorname{End}_{\mathbb{K}}\left(T_{x_{\infty}}^{\perp}\right.$ Crit $\left.\mathscr{E}\right)$ be invertible, is weaker than the coercivity condition ( (C) in Definition 2.3, namely, that $\mathscr{E}^{\prime \prime}\left(x_{\infty}\right) \xi^{2} \neq 0$ for all non-zero $\xi \in$ $T_{x_{\infty}}^{\perp}$ Crit $\mathscr{E}$.

Remark 2.6 (On Definition 2.3 and the hypotheses of Theorem 2.4). An example explained to me by Tomáš Bárta indicates the need for condition (b) in Definition 2.3 to hold for all $x \in$ Crit $\mathscr{E}$ and not just at the point $x_{\infty}$ in order for the conclusion of Theorem 2.4 to be valid: Choose $d=2$, $N=3$, and $\mathscr{E}(x, y)=x^{3}+x^{2} y^{5}$, so Crit $\mathscr{E}$ is the $y$-axis, and consider the gradient inequality at points $\left(-\frac{2}{3} y^{5}, y\right)$ in $\mathbb{K}^{2}$.

Remark 2.7 (Comparison of Theorem 2.4 and a theorem due to Huang). Huang states a result [68, Theorem 2.4.3] with a conclusion similar to that of Theorem 2.4 (albeit in a Banach-space setting), but his hypotheses are quite different than those of Theorem 2.4 and his result is better viewed as an extension of his [68, Proposition 2.7.1]. On the one hand, our condition (b) in Definition 2.3 is replaced in [68, Theorem 2.4.3] by his less restrictive condition that $\mathscr{E}^{(n)}\left(x_{\infty}\right)=0$ for $1 \leq n \leq N-1$; on the other hand, our condition (ㄷ) in Definition 2.3 is replaced in [68, Theorem 
2.4.3] by his condition that $\mathscr{E}^{(N)}\left(x_{\infty}\right) v^{N} \neq 0$ for all nonzero $v \in \operatorname{Ker} \mathscr{E}^{\prime \prime}(0)$. Our condition (国) that Crit $\mathscr{E}$ be a $C^{N}$ submanifold of $U$ is not assumed by Huang in his [68, Theorem 2.4.3].

There are other extensions of the concept of a Morse-Bott function, notably that of Kirwan [71; Holm and Karshon provide a version of her definition of a Morse-Bott-Kirwan function in [66, Definitions 2.1 and 2.3] and explore its properties and applications to topology. However, it is unclear whether the relatively simple proofs of Theorems 2.1 or 2.4 would extend to include such Morse-Bott-Kirwan functions.

2.2. Kojasiewicz gradient inequalities for generalized Morse-Bott functions. The proof of Theorem 2.1 is similar to (and also simpler than) that of Theorem 2.4 and can be obtained in [36], so we shall confine our attention to the following proof.

Proof of Theorem 2.4. We begin with several reductions that simplify the proof. First, observe that if $\mathscr{E}_{0}: U \rightarrow \mathbb{K}$ is defined by $\mathscr{E}_{0}(x):=\mathscr{E}\left(x+x_{\infty}\right)$, then $\mathscr{E}_{0}^{\prime}(0)=0$, so we may assume without loss of generality that $x_{\infty}=0$ and relabel $\mathscr{E}_{0}$ as $\mathscr{E}$. Second, let $K:=T_{x_{\infty}}$ Crit $\mathscr{E} \subset \mathbb{K}^{d}$ and observe that by noting the invariance of the conditions in Definition 2.3 under $C^{N}$ diffeomorphisms and applying a $C^{N}$ diffeomorphism to a neighborhood of the origin in $\mathbb{K}^{d}$ and possibly shrinking $U$, we may assume without loss of generality that $U \cap \operatorname{Crit} \mathscr{E}=U \cap K$, recalling that Crit $\mathscr{E} \subset U$ is a submanifold by the hypothesis that $\mathscr{E}$ is generalized Morse-Bott of order $N$ at $x_{\infty}$. Third, observe that if $\mathscr{E}_{0}: U \rightarrow \mathbb{K}$ is defined by $\mathscr{E}_{0}(x):=\mathscr{E}(x)-\mathscr{E}(0)$, then $\mathscr{E}_{0}(0)=0$, so we may once again relabel $\mathscr{E}$ as $\mathscr{E}$ and assume without loss of generality that $\mathscr{E}(0)=0$.

By the second reduction above, it suffices to consider the cases where $i) U \cap \operatorname{Crit} \mathscr{E}=\left(\mathbb{K}^{c} \oplus 0\right) \cap U$, for $d \geq 2$ and $1 \leq c \leq d-1$, or $i$ i) $U \cap \operatorname{Crit} \mathscr{E}=0 \in \mathbb{K}^{d}$, for $d \geq 1$ and $c=0$. By shrinking the open subset $U \subset \mathbb{K}^{d}$ if necessary, we may assume that $U$ is convex. Applying the Taylor Formula [80, p. 349] to a $C^{M}$ function $f: U \rightarrow \mathbb{K}^{k}$ (for $k \geq 1$ ) and integer $M \geq 1$ gives

$$
\begin{aligned}
f(x)=f( & \left.x_{0}\right)+f^{\prime}\left(x_{0}\right)\left(x-x_{0}\right)+\cdots+\frac{f^{(M-1)}\left(x_{0}\right)}{(M-1) !}\left(x-x_{0}\right)^{M-1} \\
& +\frac{1}{(M-1) !} \int_{0}^{1}(1-t)^{M-1} f^{M}\left(x_{0}+t\left(x-x_{0}\right)\right)\left(x-x_{0}\right)^{M} d t, \quad \text { for all } x, x_{0} \in U .
\end{aligned}
$$

For $i) d \geq 2$ and $0 \leq c \leq d-2$, consider

$$
v \in S^{d-1-c}=\left\{x \in \mathbb{K}^{d}: c=0 \text { or } x_{i}=0 \text { for } 1 \leq i \leq c \text { and } x_{c+1}^{2}+\cdots+x_{d}^{2}=1\right\},
$$

and for $i i) d \geq 1$ and $c=d-1$, consider $v=1$. If $\mathscr{E}$ is constant in an open neighborhood of $0 \in \mathbb{K}^{d}$, then (2.2) obviously holds, so we may assume without loss of generality that $\mathscr{E}$ is non-constant in an open neighborhood of the origin.

By viewing $\mathscr{E}(N)(0) \in \operatorname{Hom}_{\mathbb{K}}\left(\otimes^{N} \mathbb{K}^{d}, \mathbb{K}\right)=\otimes^{N} \mathbb{K}^{d *}$ and recalling that $\mathscr{E}$ is generalized MorseBott of order $N$, there is a positive constant $\zeta$ such that

$$
\left|\mathscr{E}^{(N)}(0) v^{N}\right| \geq \zeta, \quad \text { for all } v \in S^{d-1-c} .
$$

By viewing $\mathscr{E}^{(N)}(0) \in \operatorname{Hom}_{\mathbb{K}}\left(\otimes^{N-1} \mathbb{K}^{d}, \mathbb{K}^{d *}\right)$, we note that

$$
\left\|\mathscr{E}^{(N)}(0) v^{N-1}\right\|_{\mathbb{K}^{d *}}=\max _{w \in S^{d-1}-c}\left|\mathscr{E}^{(N)}(0) v^{N-1} w\right| \geq\left|\mathscr{E}^{(N)}(0) v^{N}\right|, \quad \text { for all } v \in S^{d-1-c} .
$$

The lower bounds in (2.4) ensure that

$$
\left\|\mathscr{E}^{(N)}(0) v^{N-1}\right\|_{\mathbb{K}^{d *}} \geq \zeta, \quad \text { for all } v \in S^{d-1-c} .
$$


Choose small enough positive constants $R$ and $L$ so that the closure of the cylinder $C(R, L):=$ $\left\{\kappa+r v \in \mathbb{K}^{d}: \kappa \in K\right.$ with $\|\kappa\|_{\mathbb{K}^{d}}<L$ and $r \in[0, R)$ and $\left.v \in S^{d-1-c}\right\}$ is contained in $U$. Because $\mathscr{E}^{(n)}(\kappa)=0$ for $n=1, \ldots, N-1$ and all $\kappa \in U \cap K$, the Taylor Formula (2.3) applied to $f(x)=\mathscr{E}(x)$ with $k=1$ and $M=N$ and $x_{0}=\kappa \in B_{L} \cap K$ and $x=\kappa+r v \in C(R, L)$ gives

$$
\mathscr{E}(\kappa+r v)=\frac{r^{N}}{(N-1) !} \int_{0}^{1}(1-t)^{N-1} \mathscr{E}^{N}(\kappa+t r v) v^{N} d t
$$

or equivalently,

$$
\mathscr{E}(\kappa+r v)=\frac{r^{N}}{N !} \mathscr{E}^{N}(0) v^{N}+\frac{r^{N}}{(N-1) !} \int_{0}^{1}(1-t)^{N-1}\left(\mathscr{E}^{N}(\kappa+t r v)-\mathscr{E}^{N}(0)\right) v^{N} d t .
$$

Since $\mathscr{E}$ is $C^{N}$, we may choose $R, L \in(0,1]$ small enough that

$$
\sup _{\substack{s \in[0, R),\|\kappa\|_{\mathbb{K}^{d}}<L}}\left|\left(\mathscr{E}^{N}(\kappa+s v)-\mathscr{E}^{N}(0)\right) v^{N}\right| \leq\left|\mathscr{E}^{(N)}(0) v^{N}\right|, \quad \text { for all } v \in S^{d-1-c} .
$$

Therefore, by (2.7) and (2.8) we obtain

$$
\frac{2 r^{N}}{N !}\left|\mathscr{E}^{(N)}(0) v^{N}\right| \geq|\mathscr{E}(\kappa+r v)|, \quad \text { for all } v \in S^{d-1-c} \text { and } r \in[0, R) \text { and } \kappa \in B_{L} \cap K
$$

As $\mathscr{E}^{(n)}(\kappa) v^{n}=0$ for $n=1, \ldots, N-1$ and all $\kappa \in U \cap K$ and $v \in S^{d-1-c}$, the Taylor Formula (2.3) applied to $f(x)=\mathscr{E}^{\prime}(x)$ with $k=d$ and $M=N-1$ and $x=\kappa+r v$ and $x_{0}=\kappa$ yields

$$
\mathscr{E}^{\prime}(\kappa+r v)=\frac{r^{N-1}}{(N-2) !} \int_{0}^{1}(1-t)^{N-2} \mathscr{E}^{N}(\kappa+t r v) v^{N-1} d t
$$

or equivalently,

$$
\begin{aligned}
& \mathscr{E}^{\prime}(\kappa+r v)=\frac{\mathscr{E}^{(N)}(0) v^{N-1}}{(N-1) !} r^{N-1} \\
&+\frac{r^{N-1}}{(N-2) !} \int_{0}^{1}(1-t)^{N-2}\left(\mathscr{E}^{N}(\kappa+t r v)-\mathscr{E}^{(N)}(0)\right) v^{N-1} d t, \\
& \quad \text { for all } v \in S^{d-1-c} \text { and } r \in[0, R) \text { and } \kappa \in B_{L} \cap K .
\end{aligned}
$$

Since $\mathscr{E}$ is $C^{N}$, we may choose $R, L \in(0,1]$ small enough that

$$
\sup _{\substack{s \in[0, R),\|\kappa\|_{\mathbb{K}^{d}}<L}}\left\|\left(\mathscr{E}^{N}(\kappa+s v)-\mathscr{E}^{(N)}(0)\right) v^{N-1}\right\|_{\mathbb{K}^{d *}} \leq \frac{1}{2}\left\|\mathscr{E}^{(N)}(0) v^{N-1}\right\|_{\mathbb{K}^{d *}}, \quad \text { for all } v \in S^{d-1-c}
$$

Therefore, by (2.10) and (2.11),

$$
\left\|\mathscr{E}^{\prime}(\kappa+r v)\right\|_{\mathbb{K}^{d *}} \geq \frac{r^{N-1}}{2(N-1) !}\left\|\mathscr{E}^{(N)}(0) v^{N-1}\right\|_{\mathbb{K}^{d *}}
$$

for all $v \in S^{d-1-c}$ and $r \in[0, R)$ and $\kappa \in B_{L} \cap K$. 
We compute that, for all $v \in S^{d-1-c}$ and $r \in[0, R)$ and $\kappa \in B_{L} \cap K$,

$$
\begin{aligned}
\left\|\mathscr{E}^{\prime}(\kappa+r v)\right\|_{\mathbb{K}^{d *}} \geq & \frac{r^{N-1}}{2(N-1) !}\left\|\mathscr{E}^{(N)}(0) v^{N-1}\right\|_{\mathbb{K}^{d *}} \quad(\text { by }(\underline{2.12})) \\
= & \frac{N}{4} \frac{2}{N !}\left\|\mathscr{E}^{(N)}(0) v^{N-1}\right\|_{\mathbb{K}^{d *}}\left(\frac{2}{N !}\left\|\mathscr{E}^{(N)}(0) v^{N-1}\right\|_{\mathbb{K}^{d *}}\right)^{-(N-1) / N} \\
& \times\left(\frac{2 r^{N}}{N !}\left\|\mathscr{E}(N)(0) v^{N-1}\right\|_{\mathbb{K}^{d *}}\right)^{(N-1) / N} \\
= & \frac{N}{4}\left(\frac{2}{N !}\left\|\mathscr{E}^{(N)}(0) v^{N-1}\right\|_{\mathbb{K}^{d *}}\right)^{1 / N}\left(\frac{2 r^{N}}{N !}\left\|\mathscr{E}^{(N)}(0) v^{N-1}\right\|_{\mathbb{K}^{d *}}\right)^{(N-1) / N} \\
\geq & \left.\frac{N}{4}\left(\frac{2}{N !}\left\|\mathscr{E}(N)(0) v^{N-1}\right\|_{\mathbb{K}^{d *}}\right)^{1 / N}\left(\frac{2 r^{N}}{N !}\left|\mathscr{E}^{(N)}(0) v^{N}\right|\right)^{(N-1) / N} \text { (by (2.5) }\right) \\
\geq & \left.\frac{N}{4}\left(\frac{2}{N !}\left\|\mathscr{E}(N)(0) v^{N-1}\right\|_{\mathbb{K}^{d *}}\right)^{1 / N}|\mathscr{E}(\kappa+r v)|^{(N-1) / N} \quad \text { (by (2.9) }\right) .
\end{aligned}
$$

This yields (2.2) with $\theta=(N-1) / N \in[1 / 2,1)$, for all $v \in S^{d-1-c}$ and $\kappa \in B_{L} \cap K$, and

$$
C:=\frac{N}{4} \inf _{v \in S^{d-1-c}}\left(\frac{2}{N !}\left\|\mathscr{E}^{(N)}(0) v^{N-1}\right\|_{\mathbb{K}^{d *}}\right)^{1 / N} \geq \frac{N}{4}\left(\frac{2 \zeta}{N !}\right)^{1 / N}
$$

where we apply the lower bound (2.6) to obtain the inequality in (2.13). By the reductions described earlier, this completes the proof of Theorem 2.4.

\section{3. ŁoJASIEWICZ GRADIENT INEQUALITY FOR $C^{1}$ FUNCTIONS WITH SIMPLE NORMAL CROSSINGS}

In this section, we prove Theorem 3 using a simple, coordinate-based alternative to an argument due to Bierstone and Milman of their more general [11, Theorem 2.7].

Proof of Theorem [3. By hypothesis, the function $\mathscr{E}: U \rightarrow \mathbb{K}$ has simple normal crossings in the sense of Definition 1.1 and $\mathscr{E}(0)=0$. Therefore,

$$
\mathscr{E}(x)=\mathscr{F}(x) \prod_{i=1}^{c} x_{i}^{n_{i}}, \quad \text { for all } x \in U,
$$

for integers $c \geq 1$ with $c \leq d$ and $n_{i} \geq 1$ and a $C^{1}$ function $\mathscr{F}: U \rightarrow \mathbb{K}$ with $\mathscr{F}(x) \neq 0$ for all $x \in U\left[2\right.$ Hence, if $\left\{e_{i}\right\}_{i=1}^{d}$ and $\left\{e_{i}^{*}\right\}_{i=1}^{d}$ denote the standard basis and dual basis, respectively, for $\mathbb{K}^{d}$ and $\mathbb{K}^{d *}$, then the differential of $\mathscr{E}$ is given by

$$
\begin{aligned}
\mathscr{E}^{\prime}(x) & =\sum_{j=1}^{d} \mathscr{E}_{x_{j}}(x) e_{j}^{*} \\
& =\sum_{j=1}^{c}\left(x_{j}^{n_{j}} \mathscr{F}_{x_{j}}(x)+n_{j} x_{j}^{n_{j}-1} \mathscr{F}(x)\right) \prod_{\substack{i=1 \\
i \neq j}}^{c} x_{i}^{n_{i}} e_{j}^{*}+\prod_{i=1}^{c} x_{i}^{n_{i}} \sum_{j=c+1}^{d} \mathscr{F}_{x_{j}}(x) e_{j}^{*},
\end{aligned}
$$

\footnotetext{
${ }^{2}$ By making a further coordinate change, one could assume that $\mathscr{F}=1$ without loss of generality but we shall omit that step.
} 
that is,

$$
\mathscr{E}^{\prime}(x)=\prod_{i=1}^{c} x_{i}^{n_{i}} \sum_{j=1}^{c}\left(x_{j} \mathscr{F}_{x_{j}}(x)+n_{j} \mathscr{F}(x)\right) x_{j}^{-1} e_{j}^{*}+\prod_{i=1}^{c} x_{i}^{n_{i}} \sum_{j=c+1}^{d} \mathscr{F}_{x_{j}}(x) e_{j}^{*}, \quad \text { for all } x \in U,
$$

where the sum over $j=c+1, \ldots, d$ is omitted if $c=d$. Observe that

$$
\left\|\mathscr{E}^{\prime}(x)\right\|_{\mathbb{K}^{d *}}^{2} \geq \prod_{i=1}^{c} x_{i}^{2 n_{i}} \sum_{j=1}^{c}\left(x_{j} \mathscr{F}_{x_{j}}(x)+n_{j} \mathscr{F}(x)\right)^{2} x_{j}^{-2}, \quad \text { for all } x \in U .
$$

Because $\mathscr{F}(0) \neq 0$ and $\mathscr{F}$ is $C^{1}$, there is a constant $\sigma \in(0,1]$ such that $B_{\sigma} \Subset U$ and

$$
\left|x_{j} \mathscr{F}_{x_{j}}(x)\right| \leq \frac{n_{j}}{2}|\mathscr{F}(x)|, \quad \text { for all } x \in B_{\sigma} \text { and } j=1, \ldots, c \text {, }
$$

and thus

$$
\left|x_{j} \mathscr{F}_{x_{j}}(x)+n_{j} \mathscr{F}(x)\right| \geq \frac{n_{j}}{2}|\mathscr{F}(x)|, \quad \text { for all } x \in B_{\sigma} \text { and } j=1, \ldots, c .
$$

Hence (3.3), noting that $n_{j} \geq 1$ for $j=1, \ldots, c$, yields the lower bound

$$
\left\|\mathscr{E}^{\prime}(x)\right\|_{\mathbb{K}^{d *}}^{2} \geq \frac{\mathscr{F}(x)^{2}}{4} \prod_{i=1}^{c} x_{i}^{2 n_{i}} \sum_{j=1}^{c} x_{j}^{-2}, \quad \text { for all } x \in B_{\sigma} .
$$

On the other hand, (3.1) gives

$$
\mathscr{E}(x)^{2}=\mathscr{F}(x)^{2} \prod_{i=1}^{c} x_{i}^{2 n_{i}}, \quad \text { for all } x \in U .
$$

Define

$$
m:=\inf _{x \in B_{\sigma}}|\mathscr{F}(x)|>0 \text { and } \quad M:=\sup _{x \in B_{\sigma}}|\mathscr{F}(x)|<\infty .
$$

Because $\mathscr{E}^{\prime}(0)=0$, we must have $c \geq 2$ or $c=1$ and $n_{1} \geq 2$ by examining the expression (3.2) for $\mathscr{E}^{\prime}(x)$ when $x=0$. If $c=1$, then $n_{1} \geq 2$ and inequalities (3.4), (3.5), and (3.6) give

$$
\left\|\mathscr{E}^{\prime}(x)\right\|_{\mathbb{K}^{d *}} \geq \frac{1}{2 m}\left|x_{1}\right|^{n_{1}-1} \quad \text { and } \quad|\mathscr{E}(x)| \leq M\left|x_{1}\right|^{n_{1}}, \quad \text { for all } x \in B_{\sigma} .
$$

Combining these inequalities yields

$$
\left\|\mathscr{E}^{\prime}(x)\right\|_{\mathbb{K}^{d *}} \geq \frac{m}{2 M^{\left(n_{1}-1\right) / n_{1}}}|\mathscr{E}(x)|^{\left(n_{1}-1\right) / n_{1}}, \quad \text { for all } x \in B_{\sigma},
$$

and hence we obtain (1.5) with $\theta=1-1 / n_{1}$ and $C_{0}=m /\left(2 M^{\theta}\right)$ if $c=1$.

For the remainder of the proof, we assume $c \geq 2$ and recall the Generalized Young Inequality,

$$
\left(\prod_{j=1}^{c} a_{j}\right)^{r} \leq r \sum_{j=1}^{c} \frac{a_{j}^{p_{j}}}{p_{j}}
$$

for constants $a_{j}>0$ and $p_{j}>0$ and $r>0$ such that $\sum_{j=1}^{c} 1 / p_{j}=1 / r$ (see Remark 3.1). For

$$
N:=\sum_{j=1}^{c} n_{j}
$$

we observe that the inequality,

$$
\prod_{j=1}^{c} x^{-2 n_{j} / N} \leq \frac{1}{N} \sum_{j=1}^{c} n_{j} x_{j}^{-2}, \quad \text { for } x_{j} \neq 0 \text { with } j=1, \ldots, c,
$$


follows from (3.7) by substituting $r=1$ and $a_{j}=x_{j}^{-2 n_{j} / N}$ (with $x_{j} \neq 0$ ) and $p_{j}=N / n_{j}$ for $j=1, \ldots, c$ in (3.7). Setting

$$
n:=\max _{1 \leq j \leq c} n_{j} \quad \text { and } \quad \theta:=1-1 / N \in[1 / 2,1)
$$

and applying (3.8) yields

$$
\prod_{i=1}^{c} x_{i}^{2 n_{i}} \sum_{j=1}^{c} x_{j}^{-2} \geq \frac{N}{n} \prod_{i=1}^{c} x_{i}^{2 n_{i}(1-1 / N)}
$$

that is,

$$
\prod_{i=1}^{c} x_{i}^{2 n_{i}} \sum_{j=1}^{c} x_{j}^{-2} \geq \frac{N}{n}\left(\prod_{i=1}^{c} x_{i}^{2 n_{i}}\right)^{\theta}, \quad \text { for all } x \in \mathbb{K}^{c}
$$

We now combine inequalities (3.4), (3.5), (3.6), and (3.9) to give

$$
\left\|\mathscr{E}^{\prime}(x)\right\|_{\mathbb{K}^{d *}}^{2} \geq \frac{m^{2} N}{4 n}\left(\prod_{i=1}^{c} x_{i}^{2 n_{i}}\right)^{\theta} \quad \text { and } \quad \mathscr{E}(x)^{2 \theta} \leq M^{2 \theta}\left(\prod_{i=1}^{c} x_{i}^{2 n_{i}}\right)^{\theta}, \quad \text { for all } x \in B_{\sigma} .
$$

Taking square roots and combining the preceding two inequalities yields (1.5) with constant $C_{0}=m \sqrt{N / n} /\left(2 M^{\theta}\right)$ if $c \geq 2$. This completes the proof of Theorem 3 ,

Remark 3.1 (Generalized Young Inequality). The inequality (3.7) may be deduced from Hardy, Littlewood, and Pólya [56, Inequality (2.5.2)],

$$
\prod_{i=1}^{c} b_{i}^{q_{i}} \leq \sum_{i=1}^{c} q_{i} b_{i}
$$

where $b_{i}>0$ and $c \geq 1$ and $q_{i}>0$ and $\sum_{i=1}^{c} q_{i}=1$. Indeed, set $a_{i}=b_{i}^{q_{i} / r}$, so $b_{i}=a_{i}^{r / q_{i}}$, and $p_{i}=r / q_{i}$ to give

$$
\prod_{i=1}^{c} a_{i}^{r} \leq \sum_{i=1}^{c} q_{i} a_{i}^{p_{i}} .
$$

But $q_{i}=r / p_{i}$ and thus

$$
\left(\prod_{i=1}^{c} a_{i}\right)^{r} \leq r \sum_{i=1}^{c} \frac{1}{p_{i}} a_{i}^{p_{i}}
$$

which is (3.7); see also [56, Section 8.3]. The inequality (3.7) is proved directly by Haraux as [48, Lemma 3.2 ] by using concavity of the logarithm function on $(0, \infty)$.

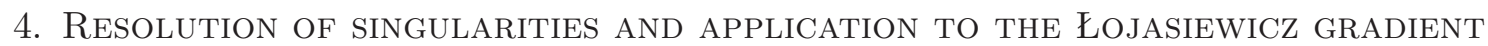 INEQUALITY}

We begin in Sections 4.1 and 4.2 by recalling the definitions of divisors and ideals, respectively, with simple normal crossings. In Section 4.3, we recall a statement of resolution of singularities for analytic varieties and in Section 4.4, we apply that to prove Theorem 1 as a corollary of Theorem 3. Unless stated otherwise, 'analytic' may refer to real or complex analytic in this section. 
4.1. Divisors with simple normal crossings. For basic methods of and notions in algebraic geometry - including blowing up, divisors, and morphisms - we refer to Griffiths and Harris [45], Hartshorne [58, and Shafarevich [110, 111. For terminology regarding real analytic varieties, we refer to Guaraldo, Macrì, and Tancredi [46]; see also Griffiths and Harris [45] and Grauert and Remmert 42 for complex analytic varieties.

Following Griffiths and Harris [45, pp. 12-14, pp. 20-22, and pp. 129-130] (who consider complex analytic subvarieties of smooth complex manifolds), let $M$ be a (real or complex) analytic (not necessarily compact) manifold of dimension $d \geq 1$ and $V \subset M$ be an analytic subvariety, that is, for each point $p \in V$, there are an open neighborhood $U \subset M$ of $p$ and a finite collection, $\left\{f_{1}, \ldots, f_{k}\right\}$ (where $k$ may depend on $p$ ), of analytic functions on $U$ such that $V \cap U=f_{1}^{-1}(0) \cap$ $\cdots \cap f_{k}^{-1}(0)$. One calls $p$ a smooth point of $V$ if $V \cap U$ is cut out transversely by $\left\{f_{1}, \ldots, f_{k}\right\}$, that is, if the $k \times d$ matrix $\left(\partial f_{i} / \partial x_{j}\right)(p)$ has rank $k$, in which case (possibly after shrinking $U$ ), we have that $V \cap U$ is an analytic (smooth) submanifold of codimension $k$ in $U$. An analytic subvariety $V \subset M$ is called irreducible if $V$ cannot be written as the union of two analytic subvarieties, $V_{1}, V_{2} \subset M$, with $V_{i} \neq V$ for $i=1,2$.

One calls $V \subset M$ an analytic subvariety of dimension $d-1$ if $V$ is a analytic hypersurface, that is, for any point $p \in V$, then $U \cap V=f^{-1}(0)$, for some open neighborhood, $U \subset M$ of $p$, and some analytic function, $f$, on $U$ [45, p. 20]. We then recall the

Definition 4.1 (Divisor on an analytic manifold). (See [45, p. 130].) A divisor D on an analytic manifold $M$ is a locally finite, formal linear combination,

$$
D=\sum_{i} a_{i} V_{i}
$$

of irreducible, analytic hypersurfaces of $M$, where $a_{i} \in \mathbb{Z}$.

We can now state the

Definition 4.2 (Simple normal crossing divisor). (See Kollár [73, Definition 3.24].) Let $X$ be a smooth algebraic variety of dimension $d \geq 1$. One says that $E=\sum_{i} E_{i}$ is a simple normal crossing divisor on $X$ if each $E_{i}$ is smooth and for each point $p \in X$ one can choose local coordinates $x_{1}, \ldots, x_{d}$ in the maximal ideal $\mathrm{m}_{p}$ of the local $\mathrm{ring}, \mathscr{O}_{p}$, of regular functions defined on some open neighborhood $U$ of $p \in X$ such that for each $i$ the following hold:

(1) Either $p \notin E_{i}$ or $E_{i} \cap U=\left\{q \in U: x_{j_{i}}(q)=0\right\}$ in an open neighborhood $U \subset X$ of $p$ for some $j_{i}$, and

(2) $j_{i} \neq j_{i^{\prime}}$ if $i \neq i^{\prime}$.

A subvariety $Z \subset X$ has simple normal crossings with $E$ if one can choose $x_{1}, \ldots, x_{d}$ as above such that in addition

(4) $Z=\left\{q \in U: x_{j_{1}}(q)=\cdots=x_{j_{s}}(q)=0\right\}$ for some $j_{1}, \ldots, j_{s}$.

In particular, $Z$ is smooth, and some of the $E_{i}$ are allowed to contain $Z$.

Kollár also gives the following, more elementary definition that serves, in part, to help compare the concepts of simple normal crossing divisor (as used by [72, 123]) and normal crossing divisor (as used by [11]), in the context of resolution of singularities.

Definition 4.3 (Simple normal crossing divisor). (See Kollár [73, Definition 1.44].) Let $X$ be a smooth algebraic variety of dimension $d \geq 1$ and $E \subset X$ a divisor. One calls $E$ a simple normal crossing divisor if every irreducible component of $E$ is smooth and all intersections are transverse. That is, for every point $p \in E$ we can choose local coordinates $x_{1}, \ldots, x_{d}$ on an open neighborhood $U \subset X$ of $p$ and $m_{i} \in \mathbb{Z} \cap[0, \infty)$ for $i=1, \ldots, d$ such that $U \cap E=\left\{q \in U: \prod_{i=1}^{d} x^{m_{i}}(q)=0\right\}$. 
Remark 4.4 (Normal crossing divisor). (See Kollár [73, Remark 1.45].) Continuing the notation of Definition 4.3, one calls $E$ a normal crossing divisor if for every $p \in E$ there are local analytic or formal coordinates, $x_{1}, \ldots, x_{d}$, and natural numbers $m_{1}, \ldots, m_{d}$ such that $U \cap E=\{q \in U$ : $\left.\prod_{i=1}^{d} x^{m_{i}}(q)=0\right\}$.

Definitions 4.2 and 4.3 extend to the categories of analytic varieties, where $\mathscr{O}_{p}$ is then the local ring of analytic functions; see, for example, Kollár [73, Section 3.44]. In the category of analytic varieties, Remark 4.4 implies that the concepts of simple normal crossing divisor and normal crossing divisor coincide 3 Definitions of simple normal crossing divisors are also provided by Cutkowsky [29, Exercise 3.13 (2)], Hartshorne [58, Remark 3.8.1] and Lazarsfeld [81, Definition 4.1.1].

4.2. Ideals with simple normal crossings. For our application to the proof of the gradient inequality, we shall need to more generally consider ideals with simple normal crossings and the corresponding statement of resolution of singularities. We review the concepts that we shall require for this purpose. For the theory of ringed spaces, sheaf theory, analytic spaces, and analytic manifolds we refer to Grauert and Remmert [43, Griffiths and Harris [45, and Narasimhan [96] in the complex analytic category and Guaraldo, Macrì, and Tancredi [46] in the real analytic category; see also Hironaka et al. [3, 4, 64. If $X$ is an analytic manifold, then $\mathscr{O}_{X}$ is the sheaf of analytic functions on $X$. An ideal $\mathscr{I} \subset \mathscr{O}_{X}$ is locally finite if for every point $p \in X$, there are an open neighborhood $U \subset X$ and a finite set of analytic functions $\left\{f_{1}, \ldots, f_{k}\right\} \subset \mathscr{O}_{U}$ such that

$$
\mathscr{I}=f_{1} \mathscr{O}_{U}+\cdots+f_{k} \mathscr{O}_{U}
$$

and $\mathscr{I}$ is locally principal if $k=1$ for each point $p \in X$.

If $p \in X$, then $\mathscr{O}_{p}$ is the ring of (germs of) analytic functions defined on some open neighborhood of $p$. The quotient sheaf $\mathscr{O}_{X} / \mathscr{I}$ is a sheaf of rings on $X$ and its support

$$
Z:=\operatorname{supp}\left(\mathscr{O}_{X} / \mathscr{I}\right)
$$

is the set of all points $p \in X$ where $\left(\mathscr{O}_{X} / \mathscr{I}\right)_{p} \neq 0$, that is, where $\mathscr{I}_{p} \neq \mathscr{O}_{p}$. In an open neighborhood $U$ of $p$ one has

$$
Z \cap U=f_{1}^{-1}(0) \cap \cdots \cap f_{k}^{-1}(0),
$$

so locally $Z$ is the zero set of finitely many analytic functions.

In order to state the version of resolution of singularities that we shall need, we recall some definitions from Cutkosky [29, pp. 40-41] and Kollár [73, Note on Terminology 3.16], given here in the real or complex analytic category, rather than the algebraic category, for consistency with our application. Suppose that $X$ is a non-singular variety and $\mathscr{I} \subset \mathscr{O}_{X}$ is an ideal sheaf; a principalization of the ideal $\mathscr{I}$ is a proper birational morphism $\pi: \widetilde{X} \rightarrow X$ such that $\widetilde{X}$ is non-singular and

$$
\pi^{*} \mathscr{I} \subset \mathscr{O}_{\widetilde{X}}
$$

is a locally principal ideal. If $X$ is a non-singular variety of dimension $d$ and $\mathscr{I} \subset \mathscr{O}_{X}$ is a locally principal ideal, then one says that $\mathscr{I}$ has simple normal crossings (or is monomial) at a point $p \in X$ if there exist local coordinates $\left\{x_{1}, \ldots, x_{d}\right\} \subset \mathscr{O}_{p}$ such that

$$
\mathscr{I}_{p}=x_{1}^{m_{1}} \cdots x_{n}^{m_{d}} \mathscr{O}_{p}
$$

for some $m_{i} \in \mathbb{Z} \cap[0, \infty)$ with $i=1, \ldots, d$. One says that $\mathscr{I}$ is locally monomial if it is monomial at every point $p \in X$ or, equivalently, if it is the ideal sheaf of a simple normal crossing divisor in the sense of Definition 4.2 .

\footnotetext{
${ }^{3} \mathrm{I}$ am grateful to Jarosław Włodarczyk for clarifying this point.
} 
Suppose that $D$ is an effective divisor on a non-singular variety $X$ of dimension $n$, so $D=$ $m_{1} E_{1}+\cdots+m_{d} E_{d}$, where $E_{i}$ are irreducible, codimension-one subvarieties of $X$, and $m_{i} \in$ $\mathbb{Z} \cap[0, \infty)$ with $i=1, \ldots, d$. One says that $D$ has simple normal crossings if

$$
\mathscr{I}_{D}=\mathscr{I}_{E_{1}}^{m_{1}} \ldots \mathscr{I}_{E_{d}}^{m_{d}}
$$

has simple normal crossings.

4.3. Resolution of singularities. We recall from Cutkosky [29, pp. 40-41] that a resolution of singularities of an algebraic or analytic variety $X$ is a proper birational morphism $\pi: \widetilde{X} \rightarrow X$ such that $\widetilde{X}$ is non-singular. Hironaka 61] proved that any algebraic variety over any field of characteristic zero admits a resolution of singularities and, moreover, that both complex and real analytic varieties admit resolutions of singularities as well [3, 4, 64, Bierstone and Milman [11] (see [12] for their expository introduction to [11]) have developed a proof of resolution of singularities that applies to real and complex analytic varieties and to algebraic varieties over any field of characteristic zero and which significantly shortens and simplifies Hironaka's proof. Additional references for resolution of singularities include Cutkowsky [29], Faber and Hauser [33], Hauser [59], Hironaka [60, Kollár [73], Villamayor [120, 121, 32], and Włodarczyk [122, 123]. Proofs of special cases of resolution of singularities for real and complex analytic varieties were previously provided by Bierstone and Milman [9, [10]. The most useful version of resolution of singularities for our application is

Theorem 4.5 (Principalization and monomialization of an ideal sheaf). (See Bierstone and Milman [11, Theorem 1.10], Kollár [73, Theorems 3.21 and 3.26 and p. 135 and Section 3.44] and Włodarczyk [123, Theorem 2.0.2] for analytic varieties; compare Włodarczyk [122, Theorem 1.0.1] for algebraic varieties.) If $X$ is a smooth analytic variety and $\mathscr{I} \subset \mathscr{O}_{X}$ is a nonzero ideal sheaf, then there are a smooth analytic variety $\tilde{X}$ and a birational and projective morphism $\pi: \tilde{X} \rightarrow X$ such that

(1) $\pi^{*} \mathscr{I} \subset \mathscr{O}_{\widetilde{X}}$ is the ideal sheaf of a simple normal crossing divisor,

(2) $\pi: \tilde{X} \rightarrow X$ is an isomorphism over $X \backslash \operatorname{cosupp} \mathscr{I}$, where $\operatorname{cosupp} \mathscr{I}\left(\operatorname{or} \operatorname{supp}\left(\mathscr{O}_{X} / \mathscr{I}\right)\right)$ is the cosupport of $\mathscr{I}$.

Versions of Theorem 4.5 when $X$ is an algebraic surface over a field of characteristic zero are provided by Cutkosky [29, p. 29] and Kollár [73, Theorem 1.74]. Kashiwara and Schapira [70] provide the following useful variant of Theorem 4.5.

Proposition 4.6 (Desingularization for the zero set of a real analytic function and its gradient map). (See Kashiwara and Schapira [70, Proposition 8.2.4].) Let $X$ be a real analytic manifold and $f: X \rightarrow \mathbb{R}$ be a real analytic function that is not identically zero on each connected component of $X$. Set $Z=\{x \in X: f(x)=0$ and $d f(x)=0\}$. Then there exists a proper morphism of real analytic manifolds $\pi: Y \rightarrow X$ that induces a real analytic diffeomorphism $Y \backslash \pi^{-1}(Z) \cong X \backslash Z$ such that, in an open neighborhood of each point $y_{0} \in \pi^{-1}(Z)$, there exist local coordinates $\left\{y_{1}, \ldots, y_{d}\right\}$ with $f \circ \pi(y)= \pm y_{1}^{n_{1}} \cdots y_{d}^{n_{d}}$, for some $n_{i} \in \mathbb{Z} \cap[0, \infty)$ with $i=1, \ldots, d$.

4.4. Application to the Eojasiewicz gradient inequality. We can now conclude the proof of one of our main theorems.

Proof of Theorem 1. As in the proof of Theorem 2.4, we may assume without loss of generality that $x_{\infty}=0$ and $\mathscr{E}(0)=0 \in \mathbb{K}$. Define $\mathscr{I}:=\mathscr{E} \mathscr{O}_{U}$ to be the ideal in $\mathscr{O}_{U}$ generated by $\mathscr{E}$, with 
support of $\mathscr{O}_{U} / \mathscr{I}$ given by $Z=\mathscr{E}^{-1}(0)$. Let $\pi: \widetilde{U} \rightarrow U$ be a resolution of singularities provided by Theorem 4.5, so

$$
\pi^{*} \mathscr{I}=\tilde{\mathscr{E}} \mathscr{O}_{\widetilde{U}}
$$

is the ideal sheaf of a simple normal crossing divisor, where $\tilde{\mathscr{E}}:=\mathscr{E} \circ \pi$ and

$$
\pi: \widetilde{U} \backslash E \cong U \backslash Z
$$

is an analytic diffeomorphism, with

$$
E:=\pi^{-1}(Z)=\{\tilde{x} \in \tilde{U}: \tilde{\mathscr{E}}(\tilde{x})=0\} \subset \widetilde{U}
$$

denoting the exceptional divisor (with ideal $\pi^{*} \mathscr{I}$ ).

By assumption, $0 \in Z$ and we may further assume without loss of generality that $0 \in \pi^{-1}(0) \subset$ $E$ and $\widetilde{U} \subset \mathbb{K}^{d}$ is an open neighborhood of the origin, possibly after shrinking $U$ and hence $\widetilde{U}$. By Theorem 4.5, the function $\tilde{\mathscr{E}}$ is the product of a monomial in the coordinate functions $x_{1}, \ldots, x_{d}$ and an analytic function $\mathscr{F}$ that is non-zero at the origin. In particular, $\tilde{\mathscr{E}}$ has simple normal crossings in the sense of Definition 1.1, possibly after further shrinking $U$ and hence $\widetilde{U}$, so $\mathscr{F}(\tilde{x}) \neq 0$ for all $\tilde{x} \in \widetilde{U}$. We can thus apply Theorem 3 to $\tilde{\mathscr{E}}=\mathscr{E} \circ \pi$ and obtain

$$
\left\|(\mathscr{E} \circ \pi)^{\prime}(\tilde{x})\right\|_{\mathbb{K}^{d *}} \geq C|(\mathscr{E} \circ \pi)(\tilde{x})|^{\theta}, \quad \text { for all } \tilde{x} \in B_{\delta},
$$

for constants $C \in(0, \infty)$ and $\theta \in[1 / 2,1)$ and $\delta \in(0,1]$. Now $(\mathscr{E} \circ \pi)(\tilde{x})=\mathscr{E}(x)$ for $x=\pi(\tilde{x}) \in U$ and therefore the preceding gradient inequality yields

$$
\left\|(\mathscr{E} \circ \pi)^{\prime}(\tilde{x})\right\|_{\mathbb{K}^{d *}} \geq C|\mathscr{E}(x)|^{\theta}, \quad \text { for all } \tilde{x} \in B_{\delta} \text { and } x=\pi(\tilde{x}) \in \pi\left(B_{\delta}\right) .
$$

The Chain Rule gives

$$
\begin{aligned}
\left\|(\mathscr{E} \circ \pi)^{\prime}(\tilde{x})\right\|_{\mathbb{K}^{d *}} & \leq\left\|\mathscr{E}^{\prime}(\pi(\tilde{x}))\right\|_{\mathbb{K}^{d *}}\left\|\pi^{\prime}(\tilde{x})\right\|_{\operatorname{End}\left(\mathbb{K}^{d}\right)} \\
& \leq M\left\|\mathscr{E}^{\prime}(\pi(\tilde{x}))\right\|_{\mathbb{K}^{d *}} \quad \text { for all } \tilde{x} \in \widetilde{U}
\end{aligned}
$$

where $M:=\sup _{\tilde{x} \in B_{\delta}}\left\|\pi^{\prime}(\tilde{x})\right\|_{\operatorname{End}\left(\mathbb{K}^{d}\right)}$. Because $\pi(\tilde{x})=x \in U$, the preceding inequality simplifies:

$$
\left\|(\mathscr{E} \circ \pi)^{\prime}(\tilde{x})\right\|_{\mathbb{K}^{d *}} \leq M\left\|\mathscr{E}^{\prime}(x)\right\|_{\mathbb{K}^{d *}}, \quad \text { for all } \tilde{x} \in \widetilde{U} \text { and } x=\pi(\tilde{x}) \in U .
$$

The map $\pi$ is open and so $\pi\left(B_{\delta}\right)$ is an open neighborhood of the origin in $\mathbb{K}^{d}$ and thus contains a ball $B_{\sigma}$ for small enough $\sigma \in(0,1]$. By combining the inequalities (4.1) and (4.2), we obtain

$$
\left\|\mathscr{E}^{\prime}(x)\right\|_{\mathbb{K}^{d *}} \geq(C / M)|\mathscr{E}(x)|^{\theta}, \quad \text { for all } x \in B_{\sigma},
$$

which is (1.1), as desired.

We can also complete the proof of one of the main corollaries.

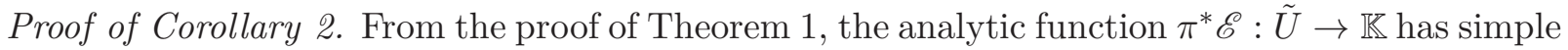
normal crossings near the origin in the sense of Definition 1.1 and so (after possibly shrinking $U$ )

$$
\pi^{*} \mathscr{E}(\tilde{x})=\mathscr{F}(\tilde{x}) \tilde{x}_{1}^{n_{1}} \cdots \tilde{x}_{d}^{n_{d}}, \quad \text { for all } \tilde{x} \in \tilde{U},
$$

where $\mathscr{F}: \tilde{U} \rightarrow \mathbb{K}$ is an analytic function such that $\mathscr{F}(\tilde{x}) \neq 0$ for all $\tilde{x} \in \tilde{U}$ and the $n_{i}$ are non-negative integers for $i=1, \ldots, d$. Theorem 3 therefore implies that $\pi^{*} \mathscr{E}$ has Eojasiewicz exponent $\theta=1-1 / N$, where $N=\sum_{i=1}^{d} n_{i}$ is the total degree of the monomial. In particular, if $\theta=1 / 2$ then $N=2$ and (after possibly relabeling the coordinates)

$$
\pi^{*} \mathscr{E}(\tilde{x})=\mathscr{F}(\tilde{x}) \tilde{x}_{1}^{2} \quad \text { or } \quad \pi^{*} \mathscr{E}(\tilde{x})=\mathscr{F}(\tilde{x}) \tilde{x}_{1} \tilde{x}_{2}, \quad \text { for all } \tilde{x} \in \tilde{U} .
$$

Hence, $\pi^{*} \mathscr{E}$ is Morse-Bott at the origin in the sense of Definition 1.2, with

$$
\text { Crit } \pi^{*} \mathscr{E}=\left\{\tilde{x} \in \tilde{U}: \tilde{x}_{1}=0\right\} \quad \text { or } \quad \text { Crit } \pi^{*} \mathscr{E}=\left\{\tilde{x} \in \tilde{U}: \tilde{x}_{1}=0 \text { and } \tilde{x}_{2}=0\right\} \text {. }
$$


From the proof of Theorem 1, the map $\pi$ is an analytic diffeomorphism from $\tilde{U} \backslash\left(\pi^{*} \mathscr{E}\right)^{-1}(0)$ onto $U \backslash \mathscr{E}^{-1}(0)$, where

$$
\operatorname{Crit} \pi^{*} \mathscr{E} \subset\left(\pi^{*} \mathscr{E}\right)^{-1}(0)=\left\{\tilde{x} \in \tilde{U}: \tilde{x}_{1}=0\right\}
$$

or

$$
\text { Crit } \pi^{*} \mathscr{E} \subset\left(\pi^{*} \mathscr{E}\right)^{-1}(0)=\left\{\tilde{x} \in \tilde{U}: \tilde{x}_{1}=0 \text { or } \tilde{x}_{2}=0\right\} .
$$

In particular, $\pi$ is an analytic diffeomorphism on the complement of a coordinate hyperplane or the union of two coordinate hyperplanes, as claimed.

\section{5. ŁoJASIEWICZ DISTANCE INEQUALITIES}

It remains to prove the distance inequalities (Corollaries 4 and 5 ). For this purpose, the proof of [11, Theorem 2.8] (see also [93]) applies but we shall include additional details for completeness. We assume a Łojasiewicz exponent $\theta \in[1 / 2,1)$, denoted by $\mu=1-\theta \in(0,1 / 2]$ in [11].

The following result on the convergence of gradient flow is a refinement of a result due to Łojasiewicz (see [89, Theorem 5] and [93, Theorem 1], where it is assumed in addition that $\mathscr{F}$ is analytic).

Theorem 5.1 (Existence and convergence of solutions to the gradient flow equation). Let $d \geq 1$ be an integer, $U \subset \mathbb{R}^{d}$ be an open subset, and $\mathscr{F}: U \rightarrow \mathbb{R}$ be a $C^{1}$ function such that $\mathscr{F}(0)=0$ and $\mathscr{F}^{\prime}(0)=0$ and $\mathscr{F} \geq 0$ on $U$ and $\mathscr{F}$ obeys the Eojasiewicz gradient inequality (1.1) with constants $C \in(0, \infty)$ and $\sigma \in(0,1]$ and $\theta \in[1 / 2,1)$ :

$$
\left\|\mathscr{F}^{\prime}(x)\right\|_{\mathbb{R}^{d *}} \geq C|\mathscr{F}(x)|^{\theta}, \quad \text { for all } x \in B_{\sigma} .
$$

Then there are a constant $\delta \in(0, \sigma / 4]$ and, for each $x \in B_{\delta}$, a solution, $\mathbf{x}$ in $\left.C\left([0, \infty) ; \mathbb{R}^{d}\right)\right) \cap$ $C^{1}\left((0, \infty) ; \mathbb{R}^{d}\right)$, to

$$
\frac{d \mathbf{x}}{d t}=-\mathscr{F}^{\prime}(\mathbf{x}(t)) \quad\left(\text { in } \mathbb{R}^{d}\right) \text { with } \mathbf{x}(0)=x,
$$

such that $\mathbf{x}(t) \in B_{\sigma / 2}$ for all $t \in[0, \infty)$ and $\mathbf{x}(t) \rightarrow \mathbf{x}_{\infty}$ in $\mathbb{R}^{d}$ as $t \rightarrow \infty$, where $\mathbf{x}_{\infty} \in B_{\sigma} \cap$ Crit $\mathscr{E}$.

Proof. When $\mathscr{F}$ is analytic (and thus $\mathscr{F}$ obeys (1.1) by Theorem 10, the conclusions were established by Łojasiewicz [93, Theorem 1]: Examination of his proof reveals that it is enough to assume that $\mathscr{F}$ obeys (1.1). The conclusions may also be obtained by specializing [34, Theorem 4] to the case of Euclidean space $\mathbb{R}^{d}$ (from the Banach and Hilbert space setting considered there) and noting that its hypotheses are fulfilled when $\mathscr{F}$ is $C^{1}$ because (1.1) holds by hypothesis here, by appealing to the Peano Existence Theorem (see Hartman [57, Theorem 2.2.1]) for its hypothesis on short-time existence of solutions to (5.1), and by appealing to the integral version [57, Equation (1.1.2)] of the gradient flow equation (5.1),

$$
\mathbf{y}(t)=\mathbf{y}(0)-\int_{0}^{t} \mathscr{F}^{\prime}(\mathbf{y}(s)) d s,
$$

for its hypothesis on estimates for $\|\mathbf{y}(t)-\mathbf{y}(0)\|_{\mathbb{R}^{d}}$ for small $t$.

We now begin the proof of one of our corollaries.

\footnotetext{
${ }^{4}$ We exclude the trivial case $\theta=1$ and $\mathscr{E}^{\prime}(0) \neq 0$.
} 
Proof of Corollary 4. Consider Item (11). Let $\delta \in(0, \sigma / 4]$ denote the constant for $\mathscr{E}$ provided by Theorem 5.1. Consider a point $x \in B_{\delta}$ such that $\mathscr{E}(x) \neq 0$ and thus $\mathscr{E}^{\prime}(x) \neq 0$ by (1.1). Let $T_{0} \in(0, \infty]$ be the smallest time such that $\mathscr{E}^{\prime}\left(\mathbf{x}\left(T_{0}\right)\right)=0$ (and thus $\mathbf{x}\left(T_{0}\right) \in B_{\sigma} \cap$ Crit $\left.\mathscr{E}\right)$, where $\mathbf{x} \in C\left([0, \infty) ; \mathbb{R}^{d}\right) \cap C^{1}\left((0, \infty) ; \mathbb{R}^{d}\right)$ is the solution to (5.1) provided by Theorem 5.1, and define the $C^{1}$ arc-length parameterization function by

$$
s(t):=\int_{0}^{t}\|\dot{\mathbf{x}}(t)\|_{\mathbb{R}^{d}} d t, \quad \text { for all } t \in\left[0, T_{0}\right),
$$

so that $d s / d t=\|\dot{\mathbf{x}}(t)\|_{\mathbb{R}^{d}}=\left\|\mathscr{E}^{\prime}(\mathbf{x}(t))\right\|_{\mathbb{R}^{d}}$ by (5.1), denoting $\dot{\mathbf{x}}(t)=d \mathbf{x} / d t$ for convenience. (We use the isometric isomorphism $\mathbb{R}^{d} \ni \xi \mapsto(\cdot, \xi)_{\mathbb{R}^{d}} \in \mathbb{R}^{d *}$ to view $\mathscr{E}^{\prime}(x)$ as an element of $\mathbb{R}^{d}$ or $\mathbb{R}^{d *}$ according to the context.) Set $S_{0}:=s\left(T_{0}\right) \in(0, \infty]$ and write $t=t(s)$ for $s \in\left[0, S_{0}\right)$. Define $\mathbf{y}(s):=\mathbf{x}(t(s))$ and observe that

$$
\frac{d \mathbf{y}}{d s}=\frac{d \mathbf{x}}{d t} \frac{d t}{d s}=\frac{d \mathbf{x}}{d t}\left(\frac{d s}{d t}\right)^{-1}=\frac{d \mathbf{x}}{d t} \frac{1}{\left\|\mathscr{E}^{\prime}(\mathbf{x}(t))\right\|_{\mathbb{R}^{d}}}=-\frac{\mathscr{E}^{\prime}(\mathbf{x}(t))}{\left\|\mathscr{E}^{\prime}(\mathbf{x}(t))\right\|_{\mathbb{R}^{d}}}, \quad \text { for all } t \in\left(0, T_{0}\right),
$$

where we again apply (5.1) to obtain the final equality. Hence, $\mathbf{y} \in C\left(\left[0, S_{0}\right) ; \mathbb{R}^{d}\right) \cap C^{1}\left(\left(0, S_{0}\right) ; \mathbb{R}^{d}\right)$ is a solution to the ordinary differential equation,

$$
\frac{d \mathbf{y}}{d s}=-\frac{\mathscr{E}^{\prime}(\mathbf{y}(s))}{\left\|\mathscr{E}^{\prime}(\mathbf{y}(s))\right\|_{\mathbb{R}^{d}}} \quad\left(\text { in } \mathbb{R}^{d}\right) \text { with } \mathbf{y}(0)=x .
$$

Write $Q(s):=\mathscr{E}(\mathbf{y}(s))$ and observe that

$$
\begin{aligned}
Q^{\prime}(s) & =\mathscr{E}^{\prime}(\mathbf{y}(s)) \mathbf{y}^{\prime}(s) \\
& =\left(\mathbf{y}^{\prime}(s), \mathscr{E}^{\prime}(\mathbf{y}(s))\right)_{\mathbb{R}^{d}} \quad(\text { inner product }) \\
& =-\frac{\left(\mathscr{E}^{\prime}(\mathbf{y}(s)), \mathscr{E}^{\prime}(\mathbf{y}(s))\right)_{\mathbb{R}^{d}}}{\left\|\mathscr{E}^{\prime}(\mathbf{y}(s))\right\|_{\mathbb{R}^{d}}}, \quad \text { for all } s \in\left[0, S_{0}\right) \quad(\text { by }(\underline{5.2})) .
\end{aligned}
$$

In particular, we obtain

$$
Q^{\prime}(s)=-\left\|\mathscr{E}^{\prime}(\mathbf{y}(s))\right\|_{\mathbb{R}^{d}}<0, \quad \text { for all } s \in\left[0, S_{0}\right) .
$$

Now $Q(0)=\mathscr{E}(x)>0$ (since $\mathscr{E} \geq 0$ on $U$ by hypothesis and $\mathscr{E}(x) \neq 0$ by assumption) and $Q(s) \leq Q(0)$ for all $s \in\left[0, S_{0}\right)$ by (5.3). But then we have

$$
\begin{aligned}
\frac{\mathscr{E}(x)^{1-\theta}}{1-\theta} & \geq \frac{Q(0)^{1-\theta}-Q(s)^{1-\theta}}{1-\theta} \\
& =-\frac{1}{1-\theta} \int_{0}^{s} \frac{d}{d u} Q(u)^{1-\theta} d u \\
& =-\int_{0}^{s} Q(u)^{-\theta} Q^{\prime}(u) d u \\
& =\int_{0}^{s} \mathscr{E}(\mathbf{y}(u))^{-\theta}\left\|\mathscr{E}^{\prime}(\mathbf{y}(u))\right\|_{\mathbb{R}^{d}} d u \\
& \geq \int_{0}^{s} C d u=C s \quad \text { for all } 0 \leq s<S_{0} \quad \text { (by (1.1) ) }
\end{aligned}
$$

In applying the Eojasiewicz gradient inequality (1.1) to obtain the last line above, we relied on the fact that $\mathbf{y}(s)=\mathbf{x}(t) \in B_{\sigma / 2}$ by (5.1) for all $t \in\left[0, T_{0}\right)$ or, equivalently, $s \in\left[0, S_{0}\right)$. Therefore,

$$
\frac{\mathscr{E}(x)^{1-\theta}}{1-\theta} \geq C S_{0}
$$


It follows that $S_{0}<\infty$ and thus as $s \uparrow S_{0}$, the solution $\mathbf{y}(s)$ converges (in $\mathbb{R}^{d}$ ) to a point $\mathbf{y}\left(S_{0}\right)=\mathbf{x}\left(T_{0}\right) \in$ Crit $\mathscr{E}$ in a finite time $S_{0}$. Moreover, by (5.1) we also have $\mathbf{x}\left(T_{0}\right) \in \bar{B}_{\sigma / 2} \subset B_{\sigma}$. Since $\left\|\mathbf{y}^{\prime}(s)\right\|_{\mathbb{R}^{d}}=1$, then $\mathbf{y}(s)$ is parameterized by arc length and

$$
\begin{aligned}
S_{0} & =\operatorname{Length}_{\mathbb{R}^{d}}\left\{\mathbf{y}(s): s \in\left[0, S_{0}\right]\right\}=\int_{0}^{S_{0}}\|\dot{\mathbf{y}}(s)\|_{\mathbb{R}^{d}} d s \\
& \geq\left\|\mathbf{y}\left(S_{0}\right)-\mathbf{y}(0)\right\|_{\mathbb{R}^{d}} \\
& =\left\|\mathbf{x}\left(T_{0}\right)-x\right\|_{\mathbb{R}^{d}} \\
& \geq \inf _{z \in B_{\sigma} \cap \operatorname{Crit} \mathscr{E}}\|z-x\|_{\mathbb{R}^{d}} \quad\left(\text { since } \mathbf{x}\left(T_{0}\right) \in B_{\sigma} \cap \text { Crit } \mathscr{E}\right) \\
& =\operatorname{dist}_{\mathbb{R}^{d}}\left(x, B_{\sigma} \cap \operatorname{Crit} \mathscr{E}\right) .
\end{aligned}
$$

From (5.4), we thus obtain

$$
\mathscr{E}(x)^{1-\theta} \geq(1-\theta) C \operatorname{dist}_{\mathbb{R}^{d}}\left(x, B_{\sigma} \cap \operatorname{Crit} \mathscr{E}\right),
$$

and this is (1.6), with exponent $\alpha=1 /(1-\theta) \in[2, \infty)$ and positive constant $C_{1}=((1-$ $\theta) C)^{1 /(1-\theta)}$.

We now assume the additional hypothesis that $B_{\sigma} \cap$ Crit $\mathscr{E} \subset B_{\sigma} \cap$ Zero $\mathscr{E}$. Hence,

$$
\begin{aligned}
\operatorname{dist}_{\mathbb{R}^{d}}\left(x, B_{\sigma} \cap \text { Crit } \mathscr{E}\right) & =\inf _{z \in B_{\sigma} \cap \text { Crit } \mathscr{E}}\|z-x\|_{\mathbb{R}^{d}} \\
& \geq \inf _{z \in B_{\sigma} \cap \text { Zero } \mathscr{E}}\|z-x\|_{\mathbb{R}^{d}} \\
& =\operatorname{dist}_{\mathbb{R}^{d}}\left(x, B_{\sigma} \cap \text { Zero } \mathscr{E}\right) .
\end{aligned}
$$

Therefore, (1.7) follows from (1.6). This proves Item (11).

Consider Item (2). We can apply (1.7) to $\mathscr{F}=\mathscr{E}^{2}$ with constants $C_{1} \in(0, \infty)$ and $\alpha=$ $1 /(1-\theta) \in[2, \infty)$ and $\sigma \in(0,1]$ and $\delta \in(0, \sigma / 4]$ determined by $\mathscr{F}$ to give

$$
\mathscr{F}(x) \geq C_{1} \operatorname{dist}_{\mathbb{R}^{d}}\left(x, B_{\sigma} \cap \text { Zero } \mathscr{F}\right)^{\alpha}, \quad \text { for all } x \in B_{\delta} .
$$

Clearly, Zero $\mathscr{E}=$ Zero $\mathscr{F}$ and therefore,

$$
\mathscr{E}(x)^{2} \geq C_{1} \operatorname{dist}_{\mathbb{R}^{d}}\left(x, B_{\sigma} \cap \text { Zero } \mathscr{E}\right)^{\alpha}, \quad \text { for all } x \in B_{\delta} .
$$

But this is (1.8), as desired, with exponent $\beta=\alpha / 2 \in[1, \infty)$ and positive constant $C_{2}=\sqrt{C_{1}}$. This completes the proof of Item (2) and hence Corollary 4 .

Next we give the proof of another corollary.

Proof of Corollary [5. We may assume without loss of generality that $x_{\infty}=0$ and $\mathscr{E}(0)=0$. Note that $B_{\sigma} \cap$ Crit $\mathscr{E} \subset B_{\sigma} \cap$ Zero $\mathscr{E}$, for small enough $\sigma \in(0,1]$, by Theorem [1. We combine the Eojasiewicz gradient and distance inequalities, (1.1) and (1.6), to give for $\delta \in(0, \sigma / 4]$ and $\alpha=1 /(1-\theta) \in[2, \infty)$,

$$
\begin{aligned}
\left\|\mathscr{E}^{\prime}(x)\right\|_{\mathbb{R}^{d *}} & \geq C_{0}|\mathscr{E}(x)|^{\theta} \\
& \geq C_{0}\left(C_{1} \operatorname{dist}_{\mathbb{R}^{d}}(x, \text { Crit } \mathscr{E})^{\alpha}\right)^{\theta}, \quad \text { for all } x \in B_{\delta} .
\end{aligned}
$$

Since $\theta \in[1 / 2,1)$, this yields (1.9) with $\mu=\alpha \theta=\theta /(1-\theta) \in[1, \infty)$ and $C_{2}=C_{0} C_{1}^{\theta}$.

Finally, we have the proof of the last corollary. 
Proof of Corollary [6. We may assume without loss of generality that $x_{\infty}=0$ and $\mathscr{E}(0)=0$. Choose $\mathscr{F}(x):=\left\|\mathscr{E}^{\prime}(x)\right\|_{\mathbb{R}^{d *}}^{2}$ for all $x \in U$ and observe that $\mathscr{F}: U \rightarrow \mathbb{R}$ is analytic and $\mathscr{F}(0)=0$, so Item (2) of Corollary 4 applies to $\mathscr{F}$ by Remark 1.4. Applying (1.8) with $\mathscr{F}$ in place of $\mathscr{E}$ gives

$$
|\mathscr{F}(x)| \geq C_{2} \operatorname{dist}_{\mathbb{R}^{d}}\left(x, B_{\sigma_{1}} \cap \text { Zero } \mathscr{F}\right)^{\alpha_{1}}, \quad \text { for all } x \in B_{\delta_{1}},
$$

for some $C_{2} \in(0, \infty)$ and $\alpha_{1} \in[1, \infty)$ and $\sigma_{1} \in(0,1]$ and $\delta_{1} \in\left(0, \sigma_{1} / 4\right]$. Since Zero $\mathscr{F}=$ Crit $\mathscr{E}$, this gives

$$
\left\|\mathscr{E}^{\prime}(x)\right\|_{\mathbb{R}^{d *}}^{2} \geq C_{2} \operatorname{dist}_{\mathbb{R}^{d}}\left(x, B_{\sigma_{1}} \cap \text { Crit } \mathscr{E}\right)^{\alpha_{1}}, \quad \text { for all } x \in B_{\delta_{1}},
$$

and taking square roots yields (1.10) with $\gamma=\alpha_{1} / 2 \in[1 / 2, \infty)$ and $C_{3}=\sqrt{C_{2}}$.

\section{Appendix A. Resolution of Singularities for the CUSP CURVE AND Bounds for its ŁOJASIEWICZ EXPONENT}

Let $\mathbb{K}=\mathbb{R}$ or $\mathbb{C}$ and recall that the cusp curve, defined as the set of solutions $(x, y) \in \mathbb{K}^{2}$ to

$$
f(x, y):=x^{2}-y^{3}=0
$$

is an elementary example used in many texts on algebraic geometry to illustrate applications of resolution of singularities. For example, see Hauser [59, Figure 10, p. 333] for a discussion and illustrations for this example and Smith [115, Section 5] or Smith, Kahanpää, Kekäläinen, and Traves [116, Chapter 7]. Our purpose in this Appendix 5 is to illustrate the use of resolution of singularities (via repeated blow ups) for $f$ on a neighborhood of the origin $0 \in \mathbb{K}^{2}$ to achieve a simple normal crossing function $\Pi^{*} f$, as predicted by Theorem 4.5. Our exposition of resolution of singularities for this example closely follows that of [115, Section 5].

Let $X:=\mathbb{K}^{2}$, and $\mathbb{P}^{1}$ be the one-dimensional projective space of all lines $\ell \subset \mathbb{K}^{2}$, and $Z:=$ $\left\{(x, y) \in \mathbb{K}^{2}: x^{2}-y^{3}=0\right\} \subset X$ and let

$$
Y=\left\{(p, \ell) \in \mathbb{K}^{2} \times \mathbb{P}^{1}: p \in \ell\right\}
$$

be the blow-up of $\mathbb{K}^{2}$ at the origin (a smooth algebraic variety of dimension two), where $\pi$ : $Y \ni(p, \ell) \mapsto p \in \mathbb{K}^{2}$ is the natural projection, $E:=\pi^{-1}(0)$ is the exceptional divisor, and $\pi: Y \backslash E \rightarrow \mathbb{K}^{2} \backslash\{0\}$ is an analytic diffeomorphism. One can show that $Y=\{(x, y,[s, t]) \in$ $\left.\mathbb{K}^{2} \times \mathbb{P}^{1}: x t-y s=0\right\}$ (for example, see [115, Lemma 5.1]). Let $U_{1}, U_{2} \subset \mathbb{P}^{1}$ denote the coordinate patches given by $U_{1}:=\{[s, t]: s \neq 0\}$ with local coordinate $z=t / s$ and $U_{2}:=\{[s, t]: t \neq 0\}$ with local coordinate $w=s / t$. Define $W_{1}:=\mathbb{K}^{2} \times U_{1}=\mathbb{K}^{3}$ and $W_{2}:=\mathbb{K}^{2} \times U_{2}=\mathbb{K}^{3}$. In the chart $W_{1}$ with coordinates $(x, y, z)$, we have $Y \cap W_{1}=\{x z-y=0\}$ and the map

$$
\phi_{1}: \mathbb{K}^{2} \ni(x, z) \mapsto(x, x z, z) \in Y
$$

identifies the coordinate neighborhood $Y \cap W_{1}$ with $\mathbb{K}^{2}$; in the chart $W_{2}$ with coordinates $(x, y, w)$, we have $Y \cap W_{2}=\{x z-y=0\}$ and the map

$$
\phi_{2}: \mathbb{K}^{2} \ni(w, y) \mapsto(w y, y, w) \in Y
$$

identifies the coordinate neighborhood $Y \cap W_{2}$ with $\mathbb{K}^{2}$. On the overlaps, we have $z=y / x$ and $w=z^{-1}=x / y$. A convenient representation of local coordinates for $Y$ is $\{x, y / x\}$ in one chart and $\{x / y, y\}$ in the other.

We shall describe the blow-up map $\pi: Y \rightarrow \mathbb{K}^{2}$ in these local coordinates. We view $Y$ as the union of two copies of $\mathbb{K}^{2}$, one with coordinates $\{x, z\}$ and the other with coordinates $\{w, y\}$, where $z=w^{-1}=y / x$. Then the pullbacks of $\pi$ by the local coordinate charts $\phi_{1}, \phi_{2}$ are given by

$$
\pi_{1}: \mathbb{K}^{2} \ni(x, z) \mapsto(x, x z) \in \mathbb{K}^{2},
$$

\footnotetext{
${ }^{5} \mathrm{I}$ am grateful to Peter Kronheimer for suggesting this example.
} 
in the first chart, and

$$
\pi_{2}: \mathbb{K}^{2} \ni(w, y) \mapsto(w y, y) \in \mathbb{K}^{2},
$$

in the second. In these coordinates, the exceptional divisor is given by $\{x=0\}$ in the first chart and by $\{y=0\}$ in the second.

We now describe the sequence of three blow ups required to achieve the monomialization $\Pi^{*} f$ of $f(x, y)=x^{2}-y^{3}$ :

(1) Use $(u, v) \mapsto(x, y)=(u v, v)$ to get $Z_{1}^{\prime}=\left\{u^{2} v^{2}-v^{3}=0\right\}$ with exceptional divisor $\{v=0\}$. Note that in this local chart for $Y$, the pull-back of the blow-up map $\pi_{1}: \mathbb{K}^{2} \rightarrow \mathbb{K}^{2}$ is not surjective since the line $\{y=0\}$ (aside from $(x, y)=(0,0))$ is not in the image. In the other local coordinate chart for $Y$, the pull-back of the blow-up map $\pi_{2}: \mathbb{K}^{2} \rightarrow \mathbb{K}^{2}$ is given by $(a, b) \mapsto(x, y)=(a, a b)$; this map is not surjective either since the line $\{x=0\}$ (aside from $(x, y)=(0,0))$ is not in the image. However, the combined blow-up map $\pi: Y \rightarrow \mathbb{K}^{2}$ is surjective. In the second coordinate chart, we have $Z_{2}^{\prime}=\left\{a^{2}-a^{3} b^{3}=0\right\}$ with exceptional divisor $\{a=0\}$.

(2) Use $(r, s) \mapsto(u, v)=(r, r s)$ to get $Z_{1}^{\prime \prime}=\left\{r^{4} s^{2}-r^{3} s^{3}=0\right\}$ with transform of the old exceptional divisor $\{s=0\}$ and exceptional divisor $\{r=0\}$. In the second coordinate chart, $(c, d) \mapsto(a, b)=(c d, d)$, we get $Z_{2}^{\prime \prime}=\left\{c^{2} d^{2}-c^{3} d^{6}=0\right\}$.

(3) Use $(\alpha, \beta) \mapsto(r, s)=(\alpha, \alpha \beta)$ to get $Z_{1}^{\prime \prime \prime}=\left\{\alpha^{6} \beta^{2}-\alpha^{6} \beta^{3}=0\right\}=\left\{\alpha^{6} \beta^{2}(1-\beta)=0\right\}$, with transform of old exceptional divisor $\{\beta=0\}$ and exceptional divisor $\{\alpha=0\}$. In the second coordinate chart, $(g, h) \mapsto(c, d)=(g h, h)$, we get $Z_{2}^{\prime \prime \prime}=\left\{g^{2} h^{4}-g^{3} h^{9}=0\right\}=$ $\left\{g^{2} h^{4}\left(1-g h^{5}\right)=0\right\}$.

Near $(\alpha, \beta)=(0,0)$, we have $Z^{\prime \prime \prime}=\left\{f_{0}(\alpha, \beta) \alpha^{6} \beta^{2}=0\right\}$ with $f_{0}(\alpha, \beta)=1-\beta$. The composition of the preceding changes of variables, $\Pi$, defines an analytic map on $\mathbb{K}^{2}$ that restricts to a diffeomorphism onto its image,

$$
\Pi: B \backslash \Pi^{-1}(Z) \ni(\alpha, \beta) \mapsto(x, y)=\left(\alpha^{3} \beta, \alpha^{2} \beta\right) \in U \backslash Z,
$$

for the open unit ball $B$ centered at the origin and an open neighborhood $U$ of the origin, where $\Pi^{*} f(\alpha, \beta)=f_{0}(\alpha, \beta) \alpha^{6} \beta^{2}$ and $\Pi^{-1}(Z)=\{\alpha=0$ or $\beta=0\}$. For $(x, y) \notin Z$, then $(\alpha, \beta)=$ $\left(x / y, y^{3} / x^{2}\right)$; the line $\{\beta=1\}$ corresponds to $\left\{x^{2}-y^{3}=0\right\}$. We may remove the factor $f_{0}$ by further choosing $\delta=\beta \sqrt{1-\beta}$ near $\beta=0$ to give

$$
\Pi^{*} f(\alpha, \delta)=\alpha^{6} \delta^{2}
$$

a monomial of total degree $N=8$. According to Theorem 3 , the monomial $\alpha^{6} \delta^{2}$ has Łojasiewicz exponent $1-1 / N=7 / 8$ and so by the last step of the proof of Theorem 1 in Section 4.4, the Łojasiewicz exponent $\theta$ of $f$ obeys $1 / 2 \leq \theta \leq 7 / 8$.

Near $(\alpha, \beta)=(0,1)$, that is, near $(\alpha, \gamma)=(0,0)$ when $\gamma=1-\beta$, we have $Z^{\prime \prime \prime}=\left\{f_{0}(\alpha, \gamma) \alpha^{6} \gamma=\right.$ $0\}$, with $f_{0}(\alpha, \gamma)=(1-\gamma)^{2}$. The composition of the preceding changes of variables defines an analytic map on $\mathbb{K}^{2}$ that restricts to a diffeomorphism onto its image,

$$
\varpi: B \backslash \varpi^{-1}(Z) \ni(\alpha, \gamma) \mapsto(x, y)=\left(\alpha^{3}(1-\gamma), \alpha^{2}(1-\gamma)\right) \in V \backslash Z,
$$

for an open neighborhood $V$ of the origin, where $\varpi^{*} f(\alpha, \gamma)=f_{0}(\alpha, \gamma) \alpha^{6} \gamma$ and $\varpi^{-1}(Z)=\{\alpha=$ 0 or $\gamma=1$ \}. We may remove the factor $f_{0}$ by further choosing $\eta=\gamma(1-\gamma)^{2}$ near $\gamma=0$ to give

$$
\varpi^{*} f(\alpha, \eta)=\alpha^{6} \eta,
$$

a monomial of total degree $N=7$. The monomial $\alpha^{6} \eta$ has Łojasiewicz exponent $1-1 / N=6 / 7$ and so the Łojasiewicz exponent $\theta$ of $f$ obeys $1 / 2 \leq \theta \leq 6 / 7$. This completes our example.

While the preceding example illustrates the role of blowing up, the Eojasiewicz exponent of an isolated critical point or zero can often be computed explicitly. For example, by applying 
Gwoździewicz [47, Theorem 1.3] and modifying its application in [47, Example, p. 365, and Example, p. 366], one can show that $\theta=2 / 3$. See also Krasiński, Oleksik, and Płoski [75, Proposition 2 and p. 3888 for the definition of weighted homogeneous polynomials]. Note also that the Hessian matrix of $f$ at the origin is given by

$$
\text { Hess } f(0,0)=\left(\begin{array}{ll}
2 & 0 \\
0 & 0
\end{array}\right)
$$

and so $f$ is not Morse-Bott at the origin.

\section{REFERENCES}

[1] O. M. Abderrahmane, On the Eojasiewicz exponent and Newton polyhedron, Kodai Math. J. 28 (2005), no. 1, 106-110. MR 2122194

[2] David Adams and Leon Simon, Rates of asymptotic convergence near isolated singularities of geometric extrema, Indiana Univ. Math. J. 37 (1988), 225-254. MR 963501 (90b:58046)

[3] J. M. Aroca, H. Hironaka, and J. L. Vicente, The theory of the maximal contact, Instituto "Jorge Juan" de Matemáticas, Consejo Superior de Investigaciones Cientificas, Madrid, 1975, Memorias de Matemática del Instituto "Jorge Juan", No. 29. [Mathematical Memoirs of the "Jorge Juan" Institute, No. 29]. MR 0444999

[4] J. M. Aroca, H. Hironaka, and J. L. Vicente, Desingularization theorems, Memorias de Matemática del Instituto "Jorge Juan" [Mathematical Memoirs of the Jorge Juan Institute], vol. 30, Consejo Superior de Investigaciones Científicas, Madrid, 1977. MR 480502

[5] Michael F. Atiyah, Resolution of singularities and division of distributions, Comm. Pure Appl. Math. 23 (1970), 145-150. MR 0256156

[6] David M. Austin and Peter J. Braam, Morse-Bott theory and equivariant cohomology, The Floer memorial volume, Progr. Math., vol. 133, Birkhäuser, Basel, 1995, pp. 123-183. MR 1362827 (96i:57037)

[7] Augustin Banyaga and David E. Hurtubise, A proof of the Morse-Bott lemma, Expo. Math. 22 (2004), no. 4, 365-373. MR 2075744

[8] I. N. Bernšteı̆n and S. I. Gel' fand, Meromorphy of the function $P^{\lambda}$, Funkcional. Anal. i Priložen. 3 (1969), no. 1, 84-85, in Russian; English translation in Functional Analysis and Its Applications 3 (1969), no. 1, pp. 68-69, https://doi.org/10.1007/BF01078276 MR 0247457

[9] Edward Bierstone and Pierre D. Milman, Semianalytic and subanalytic sets, Inst. Hautes Études Sci. Publ. Math. (1988), no. 67, 5-42. MR 972342 (89k:32011)

[10] Edward Bierstone and Pierre D. Milman, Uniformization of analytic spaces, J. Amer. Math. Soc. 2 (1989), no. 4, 801-836. MR 1001853

[11] Edward Bierstone and Pierre D. Milman, Canonical desingularization in characteristic zero by blowing up the maximum strata of a local invariant, Invent. Math. 128 (1997), no. 2, 207-302. MR 1440306

[12] Edward Bierstone and Pierre D. Milman, Resolution of singularities, Several complex variables (Berkeley, CA, 1995-1996), Math. Sci. Res. Inst. Publ., vol. 37, Cambridge Univ. Press, Cambridge, 1999, pp. 43-78. MR 1748600

[13] C. Bivià-Ausina, Multiplicity and Eojasiewicz exponent of generic linear sections of monomial ideals, Bull. Aust. Math. Soc. 91 (2015), no. 2, 191-201. MR 3314137

[14] C. Bivià-Ausina and S. Encinas, Eojasiewicz exponents and resolution of singularities, Arch. Math. (Basel) 93 (2009), no. 3, 225-234. MR 2540788

[15] C. Bivià-Ausina and S. Encinas, The Eojasiewicz exponent of a set of weighted homogeneous ideals, J. Pure Appl. Algebra 215 (2011), no. 4, 578-588. MR 2738373

[16] C. Bivià-Ausina and S. Encinas, Eojasiewicz exponent of families of ideals, Rees mixed multiplicities and Newton filtrations, Rev. Mat. Complut. 26 (2013), no. 2, 773-798. MR 3068619

[17] C. Bivià-Ausina and T. Fukui, Mixed Eojasiewicz exponents and log canonical thresholds of ideals, J. Pure Appl. Algebra 220 (2016), no. 1, 223-245. MR 3393458

[18] Raoul H. Bott, Nondegenerate critical manifolds, Ann. of Math. (2) 60 (1954), 248-261. MR 0064399 (16,276f)

[19] Raoul H. Bott, The stable homotopy of the classical groups, Ann. of Math. (2) 70 (1959), 313-337. MR 0110104

[20] S. Brzostowski, The Eojasiewicz exponent of semiquasihomogeneous singularities, Bull. Lond. Math. Soc. 47 (2015), no. 5, 848-852. MR 3403966 
[21] S. Brzostowski, T. Krasiński, and G. Oleksik, A conjecture on the Eojasiewicz exponent, J. Singul. 6 (2012), 124-130. MR 2971310

[22] N. T. N. Bùi and T. S. Pham, Computation of the Eojasiewicz exponent of nonnegative and nondegenerate analytic functions, Internat. J. Math. 25 (2014), no. 10, 1450092, 13. MR 3275820

[23] Ralph Chill, On the Eojasiewicz-Simon gradient inequality, J. Funct. Anal. 201 (2003), 572-601. MR 1986700 (2005c:26019)

[24] Ralph Chill, Alain Haraux, and M. A. Jendoubi, Applications of the Eojasiewicz-Simon gradient inequality to gradient-like evolution equations, Anal. Appl. (Singap.) 7 (2009), 351-372. MR 2572850 (2011a:35557)

[25] Tobias H. Colding and William P. Minicozzi, II, Eojasiewicz inequalities and applications, Surveys in Differential Geometry XIX (2014), 63-82, arXiv:1402.5087.

[26] Tobias H. Colding and William P. Minicozzi, II, Uniqueness of blowups and Łojasiewicz inequalities, Ann. of Math. (2) 182 (2015), no. 1, 221-285. MR 3374960

[27] Tobias H. Colding, William P. Minicozzi, II, and E. K. Pedersen, Mean curvature flow, Bull. Amer. Math. Soc. (N.S.) 52 (2015), no. 2, 297-333. MR 3312634

[28] T. C. Collins, A. Greenleaf, and M. Pramanik, A multi-dimensional resolution of singularities with applications to analysis, Amer. J. Math. 135 (2013), no. 5, 1179-1252. MR 3117305

[29] S. D. Cutkosky, Resolution of singularities, Graduate Studies in Mathematics, vol. 63, American Mathematical Society, Providence, RI, 2004. MR 2058431

[30] D. D'Acunto and K. Kurdyka, Explicit bounds for the Eojasiewicz exponent in the gradient inequality for polynomials, Ann. Polon. Math. 87 (2005), 51-61. MR 2208535

[31] C. De Lellis, Lojasiewicz-Simon inequality, Encyclopedia of Mathematics, Springer Verlag and the European Mathematical Society, 2013, http://www.encyclopediaofmath.org/index.php?title=Lojasiewicz-Simon_inequality\&oldid=36300.

[32] S. Encinas and O. Villamayor, Good points and constructive resolution of singularities, Acta Math. 181 (1998), no. 1, 109-158. MR 1654779

[33] E. Faber and H. Hauser, Today's menu: geometry and resolution of singular algebraic surfaces, Bull. Amer. Math. Soc. (N.S.) 47 (2010), no. 3, 373-417. MR 2651084

[34] Paul M. N. Feehan, Global existence and convergence of solutions to gradient systems and applications to Yang-Mills gradient flow, arXiv:1409.1525v4, xx+475 pages.

[35] Paul M. N. Feehan, On the Morse-Bott property of analytic functions on Banach spaces with Eojasiewicz exponent one half, Calc. Var. Partial Differential Equations, in press, arXiv:1803.11319.

[36] Paul M. N. Feehan, Optimal Eojasiewicz-Simon inequalities and Morse-Bott Yang-Mills energy functions, arXiv:1706.09349.

[37] Paul M. N. Feehan and Manousos Maridakis, Lojasiewicz-Simon gradient inequalities for analytic and MorseBott functions on Banach spaces, J. Reine Angew. Math., in press, arXiv:1510.03817v8.

[38] Paul M. N. Feehan and Manousos Maridakis, Eojasiewicz-Simon gradient inequalities for coupled YangMills energy functions, Memoirs of the American Mathematical Society, American Mathematical Society, Providence, RI, in press, arXiv:1510.03815v4.

[39] T. Fukui, Eojasiewicz type inequalities and Newton diagrams, Proc. Amer. Math. Soc. 112 (1991), no. 4, 1169-1183. MR 1065945

[40] A. M. Gabrièlov, Projections of semianalytic sets, Functional Anal. Appl. 2 (1968), no. 4, $282-291$. MR 0245831

[41] A. M. Gabrièlov, Multiplicities of Pfaffian intersections, and the tojasiewicz inequality, Selecta Math. (N.S.) 1 (1995), no. 1, 113-127. MR 1327229

[42] H. Grauert and R. Remmert, Analytische Stellenalgebren, Springer-Verlag, Berlin-New York, 1971, Unter Mitarbeit von O. Riemenschneider, Die Grundlehren der mathematischen Wissenschaften, Band 176. MR 0316742

[43] H. Grauert and R. Remmert, Coherent analytic sheaves, Grundlehren der Mathematischen Wissenschaften [Fundamental Principles of Mathematical Sciences], vol. 265, Springer-Verlag, Berlin, 1984. MR 755331

[44] M. Greenblatt, An elementary coordinate-dependent local resolution of singularities and applications, J. Funct. Anal. 255 (2008), no. 8, 1957-1994. MR 2462583

[45] P. Griffiths and J. Harris, Principles of algebraic geometry, Wiley-Interscience [John Wiley \& Sons], New York, 1978, Pure and Applied Mathematics. MR 507725 (80b:14001)

[46] F. Guaraldo, P. Macrì, and A. Tancredi, Topics on real analytic spaces, Advanced Lectures in Mathematics, Friedr. Vieweg \& Sohn, Braunschweig, 1986. MR 1013362 
[47] J. Gwoździewicz, The Eojasiewicz exponent of an analytic function at an isolated zero, Comment. Math. Helv. 74 (1999), no. 3, 364-375. MR 1710702

[48] Alain Haraux, Positively homogeneous functions and the Eojasiewicz gradient inequality, Ann. Polon. Math. 87 (2005), 165-174. MR 2208543

[49] Alain Haraux and M. A. Jendoubi, On the convergence of global and bounded solutions of some evolution equations, J. Evol. Equ. 7 (2007), 449-470. MR 2328934 (2008k:35480)

[50] Alain Haraux and M. A. Jendoubi, The convergence problem for dissipative autonomous systems, Springer Briefs in Mathematics, Springer, Cham; BCAM Basque Center for Applied Mathematics, Bilbao, 2015, Classical methods and recent advances, BCAM Springer Briefs. MR 3380967

[51] Alain Haraux, M. A. Jendoubi, and O. Kavian, Rate of decay to equilibrium in some semilinear parabolic equations, J. Evol. Equ. 3 (2003), 463-484. MR 2019030 (2004k:35187)

[52] Alain Haraux and T. S. Pham, On the gradient of quasi-homogeneous polynomials, Univ. Iagel. Acta Math. (2011), no. 49, 45-57. MR 3076842

[53] Alain Haraux and T. S. Pham, On the Eojasiewicz exponents of quasi-homogeneous functions, J. Singul. 11 (2015), 52-66. MR 3341438

[54] R. M. Hardt, Stratification of real analytic mappings and images, Invent. Math. 28 (1975), 193-208. MR 0372237

[55] R. M. Hardt, Triangulation of subanalytic sets and proper light subanalytic maps, Invent. Math. 38 (1976/77), no. 3, 207-217. MR 0454051

[56] G. H. Hardy, J. E. Littlewood, and G. Pólya, Inequalities, Cambridge University Press, Cambridge, 1952, 2nd ed.

[57] P. Hartman, Ordinary differential equations, Classics in Applied Mathematics, vol. 38, Society for Industrial and Applied Mathematics (SIAM), Philadelphia, PA, 2002, Corrected reprint of the second (1982) edition. MR 1929104 (2003h:34001)

[58] R. Hartshorne, Algebraic geometry, Springer-Verlag, New York-Heidelberg, 1977, Graduate Texts in Mathematics, No. 52. MR 0463157

[59] H. Hauser, The Hironaka theorem on resolution of singularities (or: A proof we always wanted to understand), Bull. Amer. Math. Soc. (N.S.) 40 (2003), no. 3, 323-403. MR 1978567

[60] Heisuke Hironaka, On resolution of singularities (characteristic zero), Proc. Internat. Congr. Mathematicians (Stockholm, 1962), Inst. Mittag-Leffler, Djursholm, 1963, pp. 507-521. MR 0175898

[61] Heisuke Hironaka, Resolution of singularities of an algebraic variety over a field of characteristic zero. I, II, Ann. of Math. (2) 79 (1964), 109-203; ibid. (2) 79 (1964), 205-326. MR 0199184

[62] Heisuke Hironaka, Introduction to real-analytic sets and real-analytic maps, Istituto Matematico "L. Tonelli" dell'Università di Pisa, Pisa, 1973, Quaderni dei Gruppi di Ricerca Matematica del Consiglio Nazionale delle Ricerche. MR 0477121

[63] Heisuke Hironaka, Subanalytic sets, (1973), 453-493. MR 0377101

[64] Heisuke Hironaka, Introduction to the theory of infinitely near singular points, Consejo Superior de Investigaciones Científicas, Madrid, 1974, Memorias de Matematica del Instituto "Jorge Juan", No. 28. MR 0399505

[65] Heisuke Hironaka, Stratification and flatness, (1977), 199-265. MR 0499286

[66] Tara Holm and Yael Karshon, The Morse-Bott-Kirwan condition is local, Res. Math. Sci. 3 (2016), Paper No. 25, 25. MR 3579296

[67] Lars Hörmander, On the division of distributions by polynomials, Ark. Mat. 3 (1958), 555-568. MR 0124734

[68] Sen-Zhong Huang, Gradient inequalities, Mathematical Surveys and Monographs, vol. 126, American Mathematical Society, Providence, RI, 2006. MR 2226672 (2007b:35035)

[69] S. Ji, J. Kollár, and B. Shiffman, A global Łojasiewicz inequality for algebraic varieties, Trans. Amer. Math. Soc. 329 (1992), no. 2, 813-818. MR 1046016

[70] M. Kashiwara and P. Schapira, Sheaves on manifolds, Grundlehren der Mathematischen Wissenschaften [Fundamental Principles of Mathematical Sciences], vol. 292, Springer-Verlag, Berlin, 1994, With a chapter in French by Christian Houzel, Corrected reprint of the 1990 original. MR 1299726

[71] F. C. Kirwan, Cohomology of quotients in symplectic and algebraic geometry, Mathematical Notes, vol. 31, Princeton University Press, Princeton, NJ, 1984. MR 766741

[72] J. Kollár, An effective Eojasiewicz inequality for real polynomials, Period. Math. Hungar. 38 (1999), no. 3, 213-221. MR 1756239

[73] J. Kollár, Lectures on resolution of singularities, Annals of Mathematics Studies, vol. 166, Princeton University Press, Princeton, NJ, 2007. MR 2289519 
[74] Hans-Joachim Kowalsky, Topological spaces, Academic Press, New York, 1964, translated from the German edition, Birkhäuser Verlag, Basel-Stuttgart, 1961. MR 0121768

[75] T. Krasiński, G. Oleksik, and A. Płoski, The Eojasiewicz exponent of an isolated weighted homogeneous surface singularity, Proc. Amer. Math. Soc. 137 (2009), no. 10, 3387-3397. MR 2515408

[76] Nicolaas H. Kuiper, $C^{1}$-equivalence of functions near isolated critical points, (1972), 199-218. Ann. of Math. Studies, No. 69. MR 0413161

[77] Hui Hsiung Kuo, The Morse-Palais lemma on Banach spaces, Bull. Amer. Math. Soc. 80 (1974), 363-365. MR 0334274

[78] K. Kurdyka, T. Mostowski, and A. Parusiński, Proof of the gradient conjecture of R. Thom, Ann. of Math. (2) 152 (2000), 763-792. MR 1815701 (2002d:37028)

[79] K. Kurdyka and A. Parusiński, $\mathbf{w}_{f}$-stratification of subanalytic functions and the Eojasiewicz inequality, C. R. Acad. Sci. Paris Sér. I Math. 318 (1994), no. 2, 129-133. MR 1260324

[80] Serge Lang, Real and functional analysis, third ed., Graduate Texts in Mathematics, vol. 142, Springer-Verlag, New York, 1993. MR 1216137

[81] R. Lazarsfeld, Positivity in algebraic geometry. I, Ergebnisse der Mathematik und ihrer Grenzgebiete. 3. Folge. A Series of Modern Surveys in Mathematics [Results in Mathematics and Related Areas. 3rd Series. A Series of Modern Surveys in Mathematics], vol. 48, Springer-Verlag, Berlin, 2004, Classical setting: line bundles and linear series. MR 2095471

[82] A. Lenarcik, On the Lojasiewicz exponent of the gradient of a holomorphic function, Singularities Symposium-Eojasiewicz 70 (Kraków, 1996; Warsaw, 1996), Banach Center Publ., vol. 44, Polish Acad. Sci. Inst. Math., Warsaw, 1998, pp. 149-166. MR 1677363

[83] A. Lenarcik, On the Eojasiewicz exponent of the gradient of a polynomial function, Ann. Polon. Math. 71 (1999), no. 3, 211-239. MR 1704300

[84] E. Lerman, Gradient flow of the norm squared of a moment map, Enseign. Math. (2) 51 (2005), $117-127$. MR 2154623 (2006b:53106)

[85] B. Lichtin, Estimation of Lojasiewicz exponents and Newton polygons, Invent. Math. 64 (1981), no. 3, 417429. MR 632982

[86] J.-M. Lion, Inégalité de Lojasiewicz en géométrie pfaffienne, Illinois J. Math. 44 (2000), no. 4, 889-900. MR 1804312

[87] Stanisław Łojasiewicz, Sur le problème de la division, Studia Math. 18 (1959), 87-136. MR 0107168 (21 \#5893)

[88] Stanisław Łojasiewicz, Sur le problème de la division, Rozprawy Mat. 22 (1961), 1-57. MR 0126072

[89] Stanisław Łojasiewicz, Une propriété topologique des sous-ensembles analytiques réels, Les Équations aux Dérivées Partielles (Paris, 1962), Éditions du Centre National de la Recherche Scientifique, Paris, 1963, pp. 87-89. MR 0160856 (28 \#4066)

[90] Stanisław Łojasiewicz, Triangulation of semi-analytic sets, Ann. Scuola Norm. Sup. Pisa (3) 18 (1964), 449-474. MR 0173265

[91] Stanisław Łojasiewicz, Ensembles semi-analytiques, (1965), Publ. Inst. Hautes Etudes Sci., Bures-sur-Yvette. LaTeX version by M. Coste, August 29, 2006 based on mimeographed course notes by S. Łojasiewicz, available at perso.univ-rennes1.fr/michel.coste/Lojasiewicz.pdf.

[92] Stanisław Łojasiewicz, Sur les ensembles semi-analytiques, (1971), 237-241. MR 0425152

[93] Stanisław Łojasiewicz, Sur les trajectoires du gradient d'une fonction analytique, Geometry seminars, 19821983 (Bologna, 1982/1983), Univ. Stud. Bologna, Bologna, 1984, pp. 115-117. MR 771152 (86m:58023)

[94] Stanisław Łojasiewicz, Sur la géométrie semi- et sous-analytique, Ann. Inst. Fourier (Grenoble) 43 (1993), 1575-1595. MR 1275210 (96c:32007)

[95] Stanisław Łojasiewicz and M.-A. Zurro, On the gradient inequality, Bull. Polish Acad. Sci. Math. 47 (1999), no. 2, 143-145. MR 1686676

[96] R. Narasimhan, Introduction to the theory of analytic spaces, Lecture Notes in Mathematics, No. 25, SpringerVerlag, Berlin-New York, 1966. MR 0217337

[97] Liviu I. Nicolaescu, An invitation to Morse theory, second ed., Universitext, Springer, New York, 2011. MR 2883440 (2012i:58007)

[98] M. Oka, Eojasiewicz exponents of non-degenerate holomorphic and mixed functions, arXiv:1704.06379.

[99] G. Oleksik, The Eojasiewicz exponent of nondegenerate singularities, Univ. Iagel. Acta Math. (2009), no. 47, 301-308. MR 2743702 
[100] J. Palis, Jr. and W. de Melo, Geometric theory of dynamical systems, Springer-Verlag, New York-Berlin, 1982, An introduction, Translated from the Portuguese by A. K. Manning. MR 669541

[101] T. S. Pham, The Eojasiewicz exponent of a continuous subanalytic function at an isolated zero, Proc. Amer. Math. Soc. 139 (2011), no. 1, 1-9. MR 2729065

[102] T. S. Pham, An explicit bound for the Eojasiewicz exponent of real polynomials, Kodai Math. J. 35 (2012), no. 2, 311-319. MR 2951259

[103] A. Płoski, Semicontinuity of the Lojasiewicz exponent, Univ. Iagel. Acta Math. (2010), no. 48, 103-110. MR 2882792

[104] J.-J. Risler and D. Trotman, Bi-Lipschitz invariance of the multiplicity, Bull. London Math. Soc. 29 (1997), no. 2, 200-204. MR 1425999

[105] T. Rodak and S. Spodzieja, Effective formulas for the local Eojasiewicz exponent, Math. Z. 268 (2011), no. 1-2, 37-44. MR 2805423

[106] E. H. Rothe, Morse theory in Hilbert space, Rocky Mountain J. Math. 3 (1973), 251-274, Rocky Mountain Consortium Symposium on Nonlinear Eigenvalue Problems (Santa Fe, N.M., 1971). MR 0334275

[107] Johan Råde, On the Yang-Mills heat equation in two and three dimensions, J. Reine Angew. Math. 431 (1992), 123-163. MR 1179335 (94a:58041)

[108] L. Schwartz, Division par une fonction holomorphe sur une variété analytique complexe, Summa Brasil. Math. 3 (1955), 181-209 (1955). MR 0139937

[109] L. Schwartz, Théorie des distributions à valeurs vectorielles. I, Ann. Inst. Fourier, Grenoble 7 (1957), 1-141. MR 0107812

[110] I. R. Shafarevich, Basic algebraic geometry. 1, third ed., Springer, Heidelberg, 2013, Varieties in projective space. MR 3100243

[111] I. R. Shafarevich, Basic algebraic geometry. 2, third ed., Springer, Heidelberg, 2013, Schemes and complex manifolds, Translated from the 2007 third Russian edition by Miles Reid. MR 3100288

[112] Leon Simon, Asymptotics for a class of nonlinear evolution equations, with applications to geometric problems, Ann. of Math. (2) 118 (1983), 525-571. MR 727703 (85b:58121)

[113] Leon Simon, Isolated singularities of extrema of geometric variational problems, Lecture Notes in Math., vol. 1161, Springer, Berlin, 1985. MR 821971 (87d:58045)

[114] Leon Simon, Theorems on regularity and singularity of energy minimizing maps, Lectures in Mathematics ETH Zürich, Birkhäuser, Basel, 1996. MR 1399562 (98c:58042)

[115] Karen E. Smith, Resolution of singularities, Jyvaskyla Summer School Lecture Notes 2016, Finland, http://www.math.lsa.umich.edu/ kesmith/JyvSummerSchool.html.

[116] Karen E. Smith, Lauri Kahanpää, Pekka Kekäläinen, and William Traves, An invitation to algebraic geometry, Universitext, Springer-Verlag, New York, 2000. MR 1788561

[117] S. Spodzieja, The Łojasiewicz exponent of subanalytic sets, Ann. Polon. Math. 87 (2005), $247-263$. MR 2208551

[118] S. Tan, S. S.-T. Yau, and H. Zuo, Eojasiewicz inequality for weighted homogeneous polynomial with isolated singularity, Proc. Amer. Math. Soc. 138 (2010), no. 11, 3975-3984. MR 2679619

[119] B. Teissier, Variétés polaires. I. Invariants polaires des singularités d'hypersurfaces, Invent. Math. 40 (1977), no. 3, 267-292. MR 0470246

[120] O. Villamayor, Constructiveness of Hironaka's resolution, Ann. Sci. École Norm. Sup. (4) 22 (1989), no. 1, 1-32. MR 985852

[121] O. Villamayor, Patching local uniformizations, Ann. Sci. École Norm. Sup. (4) 25 (1992), no. 6, 629-677. MR 1198092

[122] J. Włodarczyk, Simple Hironaka resolution in characteristic zero, J. Amer. Math. Soc. 18 (2005), no. 4, 779-822. MR 2163383

[123] J. Włodarczyk, Resolution of singularities of analytic spaces, Proceedings of Gökova Geometry-Topology Conference 2008, Gökova Geometry/Topology Conference (GGT), Gökova, 2009, pp. 31-63. MR 2500573

Department of Mathematics, Rutgers, The State University of New Jersey, 110 Frelinghuysen Road, Piscataway, NJ 08854-8019, United States of America

E-mail address: feehan@math.rutgers.edu 